\title{
Endotoxemia-induced endothelial fibrosis inhibition improves hypotension, tachycardia, multiple organ dysfunction syndrome, cytokine response, oxidative stress, and survival
}

\author{
Alejandro Vallejos $\mathbb{1}^{1,2} \cdot$ Pedro Olivares ${ }^{1} \cdot$ Sebastian Gatica ${ }^{1,2} \cdot$ Vicente Villegas $^{1} \cdot$ Cesar Echeverria $^{3}$. \\ Claudio Cabello-Verrugio ${ }^{1,2} \cdot$ Felipe Simon ${ }^{1,2}$
}

Received: 28 July 2018 / Revised: 18 February 2019 / Accepted: 20 February 2019 / Published online: 25 March 2019

(c) United States \& Canadian Academy of Pathology 2019

\begin{abstract}
Sepsis syndrome is the leading cause of mortality in critically ill patients admitted to intensive care. However, current therapies for sepsis treatment are unsatisfactory, and the mortality rate is still high. The main pathological characteristics observed during sepsis syndrome and endotoxemia include hypotension, tachycardia, multiple organ dysfunction syndrome (MODS), tissue damage, and cytokine and oxidative bursts. These conditions severely decrease the survival rates of endotoxemic patients. As a consequence of endotoxemia, large amounts of endotoxin circulate in the bloodstream throughout the vascular system and interact directly with endothelial cells that cover the inner wall of blood vessels. Endothelial cells exposed to lipopolysaccharides exhibit conversion to activated fibroblasts. By means of endotoxin-induced endothelial fibrosis, endothelial cells downregulate the expression of endothelial proteins and express fibrotic and ECM markers throughout endothelial protein expression reprogramming. Although endotoxin-induced endothelial fibrosis should, in theory, be detrimental to endothelial vascular function, the role of endothelial fibrosis in sepsis syndrome or endotoxemia is not known. Therefore, we employed a rat model to investigate whether the inhibition of endotoxin-induced endothelial fibrosis protects against endotoxemia and whether this inhibition increases survival. Our results show that the inhibition of endotoxin-induced endothelial fibrosis reduced both hypotension and tachycardia. Endotoxemia-induced MODS was also decreased when endothelial fibrosis was inhibited; treated rats showed normal kidney and liver function, inhibition of muscle mass wasting and normal glycemia. Liver and kidney histology was preserved, and organ fibrosis and fibrotic protein expression were reduced. Furthermore, pro-inflammatory cytokine secretion and NOX-2-mediated oxidative stress bursts were decreased when endothelial fibrosis was inhibited. Remarkably, the risk of death associated with sepsis syndrome at early and late time points was decreased when endotoxemia-induced endothelial fibrosis was inhibited, and a significant increase in survival was observed. These results reveal a potential novel treatment strategy to protect against sepsis syndrome and endotoxemia.
\end{abstract}

Supplementary information The online version of this article (https:// doi.org/10.1038/s41374-019-0237-6) contains supplementary material, which is available to authorized users.

Felipe Simon

fsimon@unab.cl

1 Facultad de Ciencias de la Vida, Universidad Andres Bello, Republica 330, 8370146 Santiago, Chile

2 Millennium Institute on Immunology and Immunotherapy, Alameda 340, 8331150 Santiago, Chile

3 Facultad de Medicina, Universidad de Atacama, Copayapu 485, 1531772 Copiapo, Chile

\section{Introduction}

Sepsis syndrome is a major cause of mortality in critically ill patients admitted to intensive care units [1,2]. Sepsis is characterized by uncontrolled activation of the immune system; activation of several types of immune cells involved in the synthesis and secretion of pro-inflammatory cytokines, such as tumor necrosis factor alpha (TNF- $\alpha$ ), interleukin-6 (IL-6), and IL-1 $\beta$ are observed. In addition, the immune system generates reactive oxygen species (ROS), principally by activating NAD(P)H oxidase [2-4]. Sepsis syndrome is caused and reinforced by endotoxemia, which is the accumulation of large amounts of endotoxin, such as lipopolysaccharide (LPS), from Gram-negative bacteria in 
the bloodstream $[2,3,5]$. During endotoxemia, LPS circulates throughout the vascular system, unavoidably interacting with the endothelial cells that cover the inner wall of blood vessels. Substantial efforts have been directed toward improving current therapies against these inflammatory pathologies. However, existing therapies for treating sepsis are insufficient, and the mortality rate is still high $[6,7]$.

Several deleterious features observed during sepsis syndrome and endotoxemia include decreased blood pressure, which is especially serious when it is resistant to treatment $[3,8,9]$. Hypotension is frequently treated with the administration of vasoconstrictors and the incorporation of fluid resuscitation to maintain stable blood pressure [1016]. However, this therapeutic strategy frequently fails to return the blood pressure to normal levels during septic shock, presumably because of endothelial and smooth muscle dysfunction, excessive nitric oxide (NO) secretion by vascular endothelial cells, and sympathetic vascular overstimulation, among other causes [10-17]. Some studies suggest that sepsis-induced hypotension generates severe hypoperfusion, which disturbs normal oxygen and glucose delivery to organs and decreases the clearance of carbon dioxide and waste molecules from tissues [18, 19]. This condition is critically enhanced by the increased vascular permeability mediated by endothelial dysfunction, which generates extensive edema and vascular fluid leak [20,21]. The consequence of these pathological events is to generate appropriate conditions that induce multiple organ dysfunction (MOD), which significantly enhances the sepsis mortality rate $[22,23]$. MOD syndrome (MODS) is a terminal stage of sepsis syndrome that alters the function of several crucial organs [19, 23, 24].

During the last decade, we and other groups have reported that endothelial cells exposed to inflammatory stimuli, such as endotoxin, pro-inflammatory cytokines, oxidative stress, and tumor growth factor-beta 1 and 2 (TGF- $\beta 1$ and 2), alter the endothelial expression pattern that converts endothelial cells into activated fibroblasts [25-32]. Endothelial cells exposed to endotoxin downregulate the expression of the endothelial proteins VE-cadherin and platelet-endothelial cell adhesion molecule 1 (PECAM-1, also called CD-31), whereas the fibroblast-specific genes $\alpha$ smooth muscle actin ( $\alpha$-SMA) and fibroblast-specific protein 1 (FSP-1) are upregulated [26, 27]. These results indicated that endotoxin induces an endothelial protein expression reprogramming by which endothelial cells lose endothelial proteins and gain fibroblast-like characteristics.

The mechanism that mediates endotoxin-induced endothelial fibrosis is widely understood. Endotoxin induces the synthesis and secretion of TGF- $\beta 1$ and TGF- $\beta 2$ via the Tolllike Receptor 4/ NAD(P)H Oxidase/Nuclear Factor- $\mathrm{kB}$ (TLR4/NOX/NF-kB) pathway [25-27, 32, 33]. Endotoxininduced TGF- $\beta$ isoform generation requires binding the
TGF- $\beta$ receptor type II (T $\beta R I I)$, which recruits T $\beta R I$ or activin receptor-like kinase 5 (ALK5). Subsequently, ALK5 phosphorylates smad-2/3, which then binds smad-4 to regulate target gene transcription and promote fibrosis $[25,32$, 34, 35]. Thus, endotoxin-induced endothelial fibrosis is completely dependent on TGF- $\beta$ secretion and ALK5 activation. In fact, the inhibition of ALK5 activity is effective at completely abolishing the endothelial fibrosis induced by endotoxin, suggesting that the inhibition of ALK5 activation is a useful approach to inhibit endotoxin-induced endothelial fibrosis $[25,32]$.

To date, despite findings suggesting that endotoxininduced endothelial fibrosis is detrimental to endothelial vascular function, the contribution of endothelial fibrosis to sepsis syndrome or endotoxemia is not known. Therefore, this study aimed to investigate, in a rat model, whether inhibition of endotoxin-induced endothelial fibrosis plays a protective role during endotoxemia by affecting the main characteristics of sepsis, such as hypotension, tachycardia, oxidative stress burst, pro-inflammatory cytokine secretion, tissue architecture, organ fibrosis, MODS, and survival.

The results obtained herein show for the first time that inhibition of endotoxin-induced endothelial fibrosis is protective against endotoxemia. Inhibition of endotoxininduced endothelial fibrosis reduced both the decreased systolic blood pressure and increased the heart rate to nearly normal levels. Endotoxemia-induced MODS was also decreased in endothelial cells when fibrosis was inhibited and treated animals showed normal kidney and liver function values, inhibition of muscle mass wasting and normal glycemic maintenance. Liver and kidney histology was preserved, and organ fibrosis was reduced. Furthermore, endotoxin-induced endothelial fibrosis inhibition decreased both pro-inflammatory cytokine secretion and oxidative stress bursts. Remarkably, endothelial fibrosis inhibition decreased the risk of death associated with sepsis syndrome at early and late stages after endotoxemia induction, thus increasing survival.

These results show a potential novel strategy to protect against sepsis syndrome and deleterious endotoxemia actions, opening novel possibilities in sepsis treatment.

\section{Materials and methods}

\section{Animals, experimental groups, and basic procedures}

Male Sprague-Dawley rats weighing from 100 to $120 \mathrm{~g}$ were used. Experimental protocols were approved by the Commission of Bioethics and Biosafety of the Universidad Andres Bello. Rats were separated into four groups: Group 1: vehicle-treated/saline-treated group; rats were subjected 
to GW-788388 vehicle solution administrated by gavage (vehicle-treated rats) and challenged with sham endotoxemia 1 day later (rats injected with saline solution) for $24 \mathrm{~h}$ $(N=8)$. Group 2: GW-788388-treated/saline-treated group; rats were subjected to the oral administration of $\mathrm{GW}$ $788388(5 \mathrm{mg} / \mathrm{kg})$ daily for 2 days by means of gavage [36]. After $24 \mathrm{~h}$ of GW-788388 administration, the rats were challenged with sham endotoxemia (saline solution) for $24 \mathrm{~h}$ and simultaneously administered the second GW-788388 dose $(N=6)$. Thus, GW-788388 was administered by gavage $24 \mathrm{~h}$ before and during endotoxemia. GW-788388 was prepared in a vehicle solution containing $4 \%$ dimethyl sulfoxide (DMSO) and $96 \%$ solution A (solution: $0.5 \%$ Hydroxypropyl Methylcellulose (HPMC), 5\% Tween 20, 20\% $\mathrm{HCl} 1 \mathrm{M}$ in $\mathrm{NaH}_{2} \mathrm{PO}_{4}$ 0.1 M) [37]. Group 3: vehicle-treated/endotoxemic group; rats were subjected to GW-788388 vehicle solution administrated by gavage (vehicle-treated rats). Endotoxemia was produced $24 \mathrm{~h}$ later by the IP injection of $20 \mathrm{mg} / \mathrm{kg}$ LPS (endotoxemic rats) for $24 \mathrm{~h}$, and the rats were simultaneously administered the second vehicle dose $(N=6)$. Group 4: GW-788388-treated/endotoxemic group; rats were subjected to the oral administration of GW-788388 ( $5 \mathrm{mg} / \mathrm{kg}$ ) daily for 2 days by means of gavage [36]. After $24 \mathrm{~h}$ of GW-788388 administration, endotoxemia was produced by the IP injection of $20 \mathrm{mg} / \mathrm{kg}$ LPS (endotoxemic rats) for $24 \mathrm{~h}$ and simultaneously administered the second dose of GW-788388 $(N=6)$. The rats were housed in individual cages. The peak of endotoxin-induced inflammation lasted by $12-24 \mathrm{~h}$, but signs of inflammation are observed until 72-96 h.

\section{Systolic blood pressure and heart rate}

To confirm the effectiveness of the generation of endotoxemia and detect changes in them as a consequence of GW788388 treatment, we measured systolic blood pressure $\left(\mathrm{P}_{\mathrm{S}}\right)$ and instantaneous heart rate $\left(\mathrm{f}_{\mathrm{H}}\right)$ in conscious animals for up to $72 \mathrm{~h}$ after saline or endotoxin treatment with a physiological recording acquisition system and a pressure tail cuff for noninvasive blood pressure recording system for rats (ML125/R), coupled with a MLT125/R pulse transducer (AD Instruments, Castle Hill, Australia). To perform the recordings of $\mathrm{P}_{\mathrm{S}}$ and $\mathrm{f}_{\mathrm{H}}$, animals were conscious and placed in a supine position. Tidal volume $\left(\mathrm{V}_{\mathrm{T}}\right)$ and instantaneous respiratory frequency $\left(f_{R}\right)$ were measured by transiently introducing (1 min) the rat head into a plastic mask connected to a respiratory flow head (MLT1L, AD Instruments) that measured the ventilatory flow $(\delta \mathrm{V} / \delta \mathrm{t})$, which was converted into $\mathrm{V}_{\mathrm{T}}$ through a volumetric differential pressure transducer. All transducers were connected to a PowerLab ${ }^{\circledR}$ 8/30 (AD Instruments), and physiological variables were instantaneously displayed through $\mathrm{Chart}^{\circledR}$ software (AD Instruments).

\section{Primary rat mesenteric endothelial cells culture}

Rat mesenteric endothelial cells (RMECs) were isolated from Sprague-Dawley rats in all experimental groups. The mesenteric artery was occluded close to the right kidney by tying it up, and then cannulated with a polyethylene tubing connected to a 21 gauge syringe. The mesentery was surgically removed and washed with sterile phosphate-buffered saline (PBS). For the enzymatic isolation of RMEC, each mesenteric artery was slowly perfused in a culture hood for 5 min with $5 \mathrm{~mL}$ of M-199 medium supplemented with $40 \mu \mathrm{L}$ Pen/Strept $(10,000 \mathrm{U} / \mathrm{mL} / 10,000 \mu \mathrm{g} / \mathrm{mL}), 20 \mu \mathrm{L}$ Fungizone $(250 \mu \mathrm{g} / \mathrm{mL})$, and $12.5 \mathrm{mg}$ collagenase type II. The cell suspension was centrifuged at $3000 \mathrm{rpm}$ for $7 \mathrm{~min}$; the pellet was reconstituted on $3 \mathrm{~mL}$ of M-199 medium supplemented with $8 \mathrm{~mL} / \mathrm{L}$ Pen/Strept $\quad(10,000 \mathrm{U} / \mathrm{mL} / 10,000 \mu \mathrm{g} / \mathrm{mL})$, $4 \mathrm{~mL} / \mathrm{L}$ Fungizone $(250 \mu \mathrm{g} / \mathrm{mL}), 10 \%$ fetal bovine serum (FBS), and $10 \%$ cosmic calf serum (CCS), and then plated on gelatin-coated wells in a six-well plate. Cells were allowed to attach to the gel matrix for $4 \mathrm{~h}$, then rinsed once with sterile PBS and finally cultured on M-199 medium supplemented with $8 \mathrm{~mL} / \mathrm{L}$ Pen/Strept $(10,000 \mathrm{U} / \mathrm{mL} /$ $10,000 \mu \mathrm{g} / \mathrm{mL}), 4 \mathrm{~mL} / \mathrm{L}$ Fungizone $(250 \mu \mathrm{g} / \mathrm{mL}), 10 \% \mathrm{FBS}$, and $10 \% \mathrm{CCS}$. The primary culture was grown at $37^{\circ} \mathrm{C}$ in a $5 \% \mathrm{CO}_{2}$ incubator for three days. For all experiments, passages used were 2-4 and confluency of cells was 70$90 \%$. Experiments were performed in serum-reduced media (1\% FBS) and cells were cultured in 1\% FBS 24-h before experiments.

\section{Endothelial cell line culture}

Human umbilical vein endothelial cell (HUVEC)-derived endothelial cell line EAhy926 was cultured at $37^{\circ} \mathrm{C}$ in a 5\%:95\% $\mathrm{CO}_{2}$ :air atmosphere in Dulbecco's modified Eagle's medium-low glucose (GIBCO, USA) supplemented with $10 \%$ FBS, $2 \mathrm{mM}$ glutamine, and $50 \mathrm{U} / \mathrm{mL}$ penicillinstreptomycin (Sigma). For all experiments, passages used were 8-10 and confluency of cells was 70-90\%. Experiments were performed in serum-reduced media (1\% FBS) and cells were cultured in 1\% FBS 24-h before experiments. For GW-788388 treatment, cultured cells were treated with $5 \mu \mathrm{g} / \mathrm{mL}$ GW-788388 $1 \mathrm{~h}$ before and during endotoxin treatment.

\section{Primary cell culture}

HUVEC were isolated by collagenase $(0.25 \mathrm{mg} / \mathrm{mL})$ digestion from freshly obtained umbilical cord veins from normal pregnancies, after patient's informed consent. The investigation conforms with the principles outlined in the Declaration of Helsinki. The Commission of Bioethics and Biosafety of Universidad Andres Bello also approved all 
experimental protocols. Cells were grown in gelatin-coated dishes at $37{ }^{\circ} \mathrm{C}$ in a $5 \%: 95 \% \mathrm{CO}_{2}$ :air atmosphere in medium 199 (Sigma, MO), containing $100 \mu \mathrm{g} / \mathrm{mL}$ endothelial cell growth supplement (ECGS) (Sigma), $100 \mu \mathrm{g} / \mathrm{mL}$ heparin, 5 $\mathrm{mM}$ D-glucose, $3.2 \mathrm{mM}$ L-glutamine, 10\% FBS (GIBCO, $\mathrm{NY}$ ), and $50 \mathrm{U} / \mathrm{mL}$ penicillin-streptomycin (Sigma). HUVECs were used between the 3rd and 5th in vitro passage and always before the 10th day in culture, and 25-30 different batches of HUVECs were used.

\section{Histological analysis using hematoxylin/eosin and picroSirius red staining}

The kidneys and livers were extracted from each experimental group $24 \mathrm{~h}$ after the challenge. Formalin-fixed, paraffin-embedded samples were sectioned and subjected to hematoxylin/eosin and picroSirius red staining. For hematoxylin/eosin staining, liver and kidney tissue damages were blindly analyzed and recorded using a tissue damage score according to the following grading scale: normal $=0$, minimal $=1$, mild $=2$, moderate $=3$, marked $=4$, and severe $=5$. The analysis criteria included the following tissue damage characteristics: liver, morphological changes, cytoplasmic vacuolation, neutrophil/polymorphonuclear infiltration and erythrocyte accumulation; and kidney, enhanced swelling of renal cells, glomerular structure alteration and swelling, vacuolar degeneration and tubular cell necrosis. For picroSirius red staining, liver and kidney (glomerular and interstitial regions) analyses were performed using ImageJ software. Fibrosis was quantified by selecting 10 observation fields from at least three different tissues under the experimental conditions. The images were divided into the red, green and blue (RGB) channels and converted to grayscale. The green channel was selected, and its threshold was calculated manually, representing the fibrotic area of the tissue.

\section{Plasma measurements of MOD markers, antioxidant molecules, oxidative stress, and pro-inflammatory cytokines}

Twenty-four hours after saline or endotoxin administration, cardiac puncture was performed for blood extraction into lithium heparin-containing tubes. The blood was immediately centrifuged at $4000 \mathrm{rpm}$ for $10 \mathrm{~min}$ at $4{ }^{\circ} \mathrm{C}$ to separate the plasma, which was then used to measure the markers of MOD, oxidative stress, glutathione (GSH), $\alpha$-tocopherol and cytokines TNF- $\alpha$, IL-1 $\beta$, IL-6, IL-10, IL-12, and IL-4. Plasma cytokines were measured at $12,24,48,72 \mathrm{~h}$ after endotoxin administration. Plasma ROS were measured in peripheral blood mononuclear cell (PBMC) fractions using the ROS-sensitive probe 2,7-dichlorofluorescin diacetate (DCFH-DA). DCFH-DA is a stable, non-fluorescent, and cell-permeable molecule that is hydrolyzed by intracellular esterases into the non-fluorescent 2,7-dichlorofluorescin $(\mathrm{DCFH})$, which is oxidized in the presence of peroxides into the highly fluorescent 2,7-dichlorofluorescein (DCF), which is detected at $488 \mathrm{~nm}$. We also used the probe dihydroethidium (DHE), which is fluorescent $(570 \mathrm{~nm})$ and preferentially interacts with the superoxide anion. Reduced GSH levels were measured from $0.2 \mathrm{ml}$ of the plasma fraction mixed with $2.25 \mathrm{ml}$ of $0.1 \mathrm{~mol} / \mathrm{L} \mathrm{K}$-phosphate buffer ( $\mathrm{pH} 8.0)$ and $25 \mu \mathrm{L}$ of Ellman's reagent $(10 \mathrm{mmol} / \mathrm{L}$ dithionitrobenzoic acid in methanol). After $1 \mathrm{~min}$, the assay absorbance was measured at $412 \mathrm{~nm}$. $\alpha$-Tocopherol levels were measured from $0.5 \mathrm{~mL}$ of the plasma fraction mixed with $1 \mathrm{~mL}$ of $\mathrm{n}$-heptane. Then, after centrifugation, the heptane phase was collected for high performance liquid chromatography (HPLC) analysis. A Supercosil-LC-Si column (Supelco, Bellefonte, PA) was used, and a $298 \mathrm{~nm}$ excitation was used to detect the fluorescence. TNF- $\alpha$, IL$1 \beta$, IL-6, IL-10, IL-12, and IL-4 were measured with an enzyme-linked immunosorbent assay (ELISA) according to the manufacturers' instructions (R\&D Systems Inc., MN, USA). To measure markers of MOD, we measured plasma levels of aspartate aminotransferase (AST), alanine aminotransferase (ALT), total bilirubin (TBIL), and gammaglutamyl transferase (GGT) for the liver; creatinine (CRE) and blood urea nitrogen (BUN) for the kidney; creatine kinase (CK) for muscle mass wasting; and glycemia (GLY) for metabolic function. MOD markers were measured with the Piccolo Xpress Chemistry Analyzer (MetLyte and General Chemistry, 13 panel) (Abaxis, CA, USA) and the iSTAT System (CG4 + cartridge) (Abbott Laboratories, IL, USA), according to the manufacturer's instructions.

\section{Endothelial and fibrotic protein detection in cultured endothelial cells and fresh primary RMECs}

Cultured endothelial cells (EAhy926) [38] were cultured in serum-reduced media (1\% FBS) $24 \mathrm{~h}$ before and during experiments. Then, cells were treated with GW-788388 $(5 \mu \mathrm{g} / \mathrm{mL})$ for $1 \mathrm{~h}$ before $24 \mathrm{~h}$ endotoxin $(20 \mu \mathrm{g} / \mathrm{mL})$ or vehicle exposure. Cultured endothelial cells (EAhy926) [38] and rat primary mesenteric endothelial cells were lysed in cold lysis buffer [150 mM NaCl, $1 \mathrm{mM}$ EGTA, $50 \mathrm{mM}$ Tris, $\mathrm{pH} 7.4,1 \%$ glycerol, $1 \%$ Triton $\mathrm{X}-100,10 \mathrm{mM} \mathrm{NaF}$, $1 \mathrm{mM}$ sodium orthovanadate $20 \mathrm{mM} \mathrm{NaPi}$, and protease inhibitor cocktail (Sigma-Aldrich, St Louis, MO, USA)], centrifuged $\left(10,000 \mathrm{~g}\right.$ for $15 \mathrm{~min}$ at $\left.4{ }^{\circ} \mathrm{C}\right)$ and the proteins were then extracted. Supernatants were collected and stored in the same lysis buffer. The protein extract and supernatant were subjected to sodium dodecyl sulfate-polyacrylamide gel electrophoresis (SDS-PAGE), and resolved proteins were transferred to a nitrocellulose or polyvinylidene difluoride (PVDF) membrane. The blocked membrane was 
incubated with the appropriate primary antibody, washed twice, and incubated with a secondary antibody. Bands were revealed using a peroxidase-conjugated $\mathrm{IgG}$ antibody. Peroxidase activity was detected through enhanced chemiluminescence (Bio-Rad, CA), and images were acquired using Fotodyne FOTO/Analyst Luminary Workstations Systems (Fotodyne, Inc., Hartland, WI). Protein content was determined by densitometric scanning of immunoreactive bands, and intensity values were obtained by densitometry of individual bands normalized against tubulin. For measurement of smad-2 phosphorylation, the anti-psmad-2 primary antibody bands were normalized against total smad-2. RMECs were isolated from rats of the four experimental groups described above. Animals were anesthetized with isofluorane and subjected to surgery. The mesenteric artery was occluded close to the right kidney by tying it up followed by cannulation with polyethylene tubing connected to a 21 gauge syringe. The mesentery was surgically removed and washed with sterile PBS. The cell suspension was centrifuged at $3000 \mathrm{rpm}$ for $7 \mathrm{~min}$; the pellet was subjected to protein extraction as described above. For a detailed list of antibodies used in western blot experiments, see Supplementary Table S1.

\section{Fluorescent immunocytochemistry and immunohistochemistry in primary endothelial cells and fresh rat blood vessels}

Fluorescent immunocytochemistry: confluent primary HUVEC cells were washed twice with PBS and fixed with $3.7 \%$ paraformaldehyde (PFA) for $30 \mathrm{~min}$ at room temperature (RT) before being permeabilized with $0.1 \%$ Triton $\mathrm{X}-100$ in PBS for 30 min at RT and then blocked for $2 \mathrm{~h}$ at RT with $3 \%$ bovine serum albumin (BSA) in PBS. The cells were subsequently washed again and incubated with the primary antibodies to detect endothelial and fibrotic proteins. Then, the cells were washed twice and incubated with the secondary antibodies. Samples were mounted with ProLong Gold antifade mounting medium with 4',6-diamidino-2-phenylindole (DAPI) (Invitrogen Life Technologies, Carlsbad, CA, USA). For a detailed list of the antibodies used, see Supplementary Table S2. For the quantification of fluorescence of VE-cadherin (red channel) and $\alpha$-SMA (green channel) in the endothelium of HUVECs, areas of interest were selected and subjected to analysis using the Image $\mathbf{J}$ software. Fluorescence quantification was normalized against control condition (vehicle-treated cells).

Fluorescent immunohistochemistry: mesenteric artery, aorta artery, and renal vein were obtained from rats of all four experimental groups described above. The animals were anesthetized with isofluorane and subjected to surgery. Vessels were extracted, fixed with PFA $3.7 \%$ for $1 \mathrm{~h}$ at RT, permeabilized with $0.1 \%$ Triton X-100 in PBS for $30 \mathrm{~min}$ at
RT, blocked for $3 \mathrm{~h}$ at RT with $3 \% \mathrm{BSA}$ in PBS, and incubated at RT for $20 \mathrm{~min}$ in $50 \mathrm{mM} \mathrm{NH}_{4} \mathrm{Cl}$ in PBS. The samples were subsequently washed and incubated with the primary antibodies to detect endothelial and fibrotic proteins. Then, the cells were washed twice and incubated with the secondary antibodies. Nuclei were stained with Hoechst (Sigma). For a detailed list of the antibodies used, see Supplementary Table S2. For the quantification of fluorescence of VE-cadherin (red channel) and fibronectin (FN; green channel) in the endothelium of mesenteric artery, aorta artery, and renal vein images, areas of interest were selected and subjected to analysis using the Image $\mathbf{J}$ software. Fluorescence quantification was normalized against control condition (vehicle-treated/saline-treated).

\section{Cell viability determination}

Cell viability was determined using the 3-(4,5-dimethylthiazol-2-yl)-2,5-diphenyltetrazolium bromide (MTT) colorimetric assay (Invitrogen Life Technologies, Carslbad, CA, USA), in which cell viability was quantified by the amount of MTT reduction. After GW-788388 treatment was performed, endothelial cells were co-incubated with anhydrous MTT $4 \mathrm{~h}$ and then solubilized with an isopropanol/ DMSO solution. The optical density value was measured at $540 \mathrm{~nm}$.

\section{RNA isolation and RT-qPCR}

Quantitative reverse transcription PCR (RT-qPCR) experiments were performed to measure tissue factor (TF) and von Willebrand factor (vWF), NAD(P)H Oxidase-1 (NOX-1), NOX-2, NOX-4 mRNA levels in primary RMEC. Total RNA was extracted with Trizol according to the manufacturer's protocol (Invitrogen, Carlsbad, CA). DNAse Itreated RNA was used for reverse transcription using the Super Script II Kit (Invitrogen, Carlsbad, CA). Equal amounts of RNA were used as templates in each reaction. Q-PCR was performed using the SYBR Green PCR Master Mix (AB Applied Biosystems, Foster City, CA). Assays were run using a Rotor-gene system (Corbet Research) instrument. Data are presented as relative mRNA levels of the gene of interest normalized to relative levels of $28 \mathrm{~S}$ mRNA.

\section{Reagents}

LPS from Escherichia coli (serotype 0127:B8) and Hoechst were purchased from Sigma-Aldrich. GW-788388 was purchased from MedChemExpress, NJ, USA. Anti-p-smad2 and total anti-smad-2 were purchased from Cell Signaling Technology (Beverly, MA.USA), Buffers and salts were purchased from Merck Biosciences. DCFH-DA and DHE 
were purchased from Invitrogen Life Technologies (Carslbad, CA, USA).

\section{Statistical analysis}

All results are presented as the mean \pm SD or mean $\pm 95 \%$ confidence interval $(\mathrm{CI})$ for the relative risk. Differences were considered significant at $p<0.05$ and $p<0.01$. Significant differences in systolic blood pressure recording experiments were assessed by one-way analysis of variance (ANOVA) followed by Dunnett's post-test to compare them with basal recordings and by two-way ANOVA followed by the Bonferroni post-test to compare the vehicle-treated/ endotoxemic group with the GW-treated/endotoxemic group recordings (see the figure legends for detailed explanations). Plasma measurements were performed by one-way ANOVA (Kruskall-Wallis) followed by Dunn's post-test. Contingency analyses with Fisher's exact test were used to assess the relative risk of death. Kaplan-Meier curves, the log-rank, and Gehan-Breslow-Wilcoxon tests were used to determine survival rates.

\section{Results}

\section{Endotoxemia-induced endothelial fibrosis inhibition improves hypotension and tachycardia during endotoxemia}

To investigate whether endothelial fibrosis induced by endotoxemia promotes detrimental actions on the control of both blood pressure and heart rate, we performed experiments in endotoxemic rats subjected to endothelial fibrosis inhibition, in which $\mathrm{P}_{\mathrm{S}}$ and $\mathrm{f}_{\mathrm{H}}$ were recorded.

In order to generate in vivo inhibition of endothelial fibrosis, rats were treated with the widely used ALK5 in vivo inhibitor, GW-788388 $(5 \mathrm{mg} / \mathrm{kg})$ administrated orally by gavage, twice a day before and during endotoxemia $[36,37,39,40]$. After $24 \mathrm{~h}$ of GW-788388 administration, endotoxemia was produced by IP injection of LPS $(20 \mathrm{mg} / \mathrm{kg})$. Endotoxemia was followed by the next $24 \mathrm{~h}$ and GW-788388 was administered throughout the experiment to maintain the endothelial fibrosis inhibition condition. Despite, GW-788388 is a well-accepted ALK5 signaling in vivo inhibitor [36, 37, 39, 40], we performed several experiments to demonstrate that under our experimental conditions GW-788388 treatment was effective to inhibit endotoxemia-induced endothelial fibrosis.

First, endothelial cell line cultures exposed to endotoxin showed significantly decreased expression of the endothelial marker VE-cadherin, whereas the expression levels of both the fibrotic protein $\alpha$-SMA and the ECM protein FN (Fig. 1a-c) were increased. However, endotoxin-exposed endothelial cells in the presence of GW-788388 failed to change the levels of the endothelial marker VE-Cadherin (Fig. 1a), as well as those of the fibrotic proteins $\alpha$-SMA (Fig. 1b) and FN (Fig. 1c), demonstrating that GW-788388 inhibited endotoxin-induced endothelial fibrosis in this in vitro model. In addition, GW-788388 did not affect cell viability in the range of concentrations used in this study (Fig. 1d). Furthermore, immunocytochemistry experiments were performed to evaluate whether GW-788388 inhibits endotoxin-induced endothelial fibrosis. To that end, primary human endothelial cells extracted from umbilical vein (primary HUVEC) were used. HUVECs exposed to vehicle exhibited VE-cadherin labeling localized predominantly at the plasma membrane, whereas $\alpha$-SMA was virtually not detected (Fig. 1e). In contrast, endothelial cells exposed to endotoxin showed reduced VE-cadherin levels and increased $\alpha$-SMA labeling as fibrotic-like stress fibers (Fig. 1f). Importantly, GW-treated endothelial cells exposed to endotoxin were resistant to the endotoxin-mediated change and showed VE-cadherin and $\alpha$-SMA labeling (Fig. 1g) similar to those depicted in cells in the presence of vehicle or GW alone (Fig. 1e, h, respectively). Concordantly, immunocytochemistry quantification performed in HUVECs exposed to endotoxin showed that VE-cadherin (Fig. 1i, left panel) and FN (Fig. 1i, right panel) fluorescence levels were decreased and increased, respectively, compared with control conditions. However, GW-treated HUVECs exposed to endotoxin failed to change VEcadherin and FN fluorescence levels (Fig. 1i). Considering that ALK5 intracellular signaling induces smad-2 phosphorylation, we were prompted to assess whether GW788388 inhibits endotoxin-induced smad-2 phosphorylation. Endotoxin-treated endothelial cells in the presence of GW-788388 failed to increase p-smad-2, whereas in the absence of GW, smad-2 phosphorylation was strong (Fig. 1j). Similar results are obtained when TGF- $\beta$ was used as an ALK5 inducer (data not shown).

Next, we tested the effectiveness of GW-788388 to inhibit endotoxemia-induced endothelial fibrosis in vivo in a model of endotoxemia induced by LPS administration. To that end, rats were treated with vehicle (vehicletreated rats) or GW-788388 (GW-treated rats), and then challenged with IP endotoxin injection (endotoxic rats) or sham endotoxemia (rats injected with saline solution). After procedure, mesenteric artery (Fig. $1 \mathrm{k}-\mathrm{n}$ ), aorta (Fig. 1o-r), and renal vein (Fig. 1s-v) were extracted and subjected immediately to immunohistochemistry. Vehicle-treated rats subjected to the saline solution IP injection (vehicle-treated/saline-treated group) showed high VE-cadherin labeling and nearly no detectable FN expression (Fig. 1k, o, s). As expected, vehicle-treated rats subjected to endotoxemia (vehicle-treated/endotoxemic group) showed a decreased VE-cadherin 

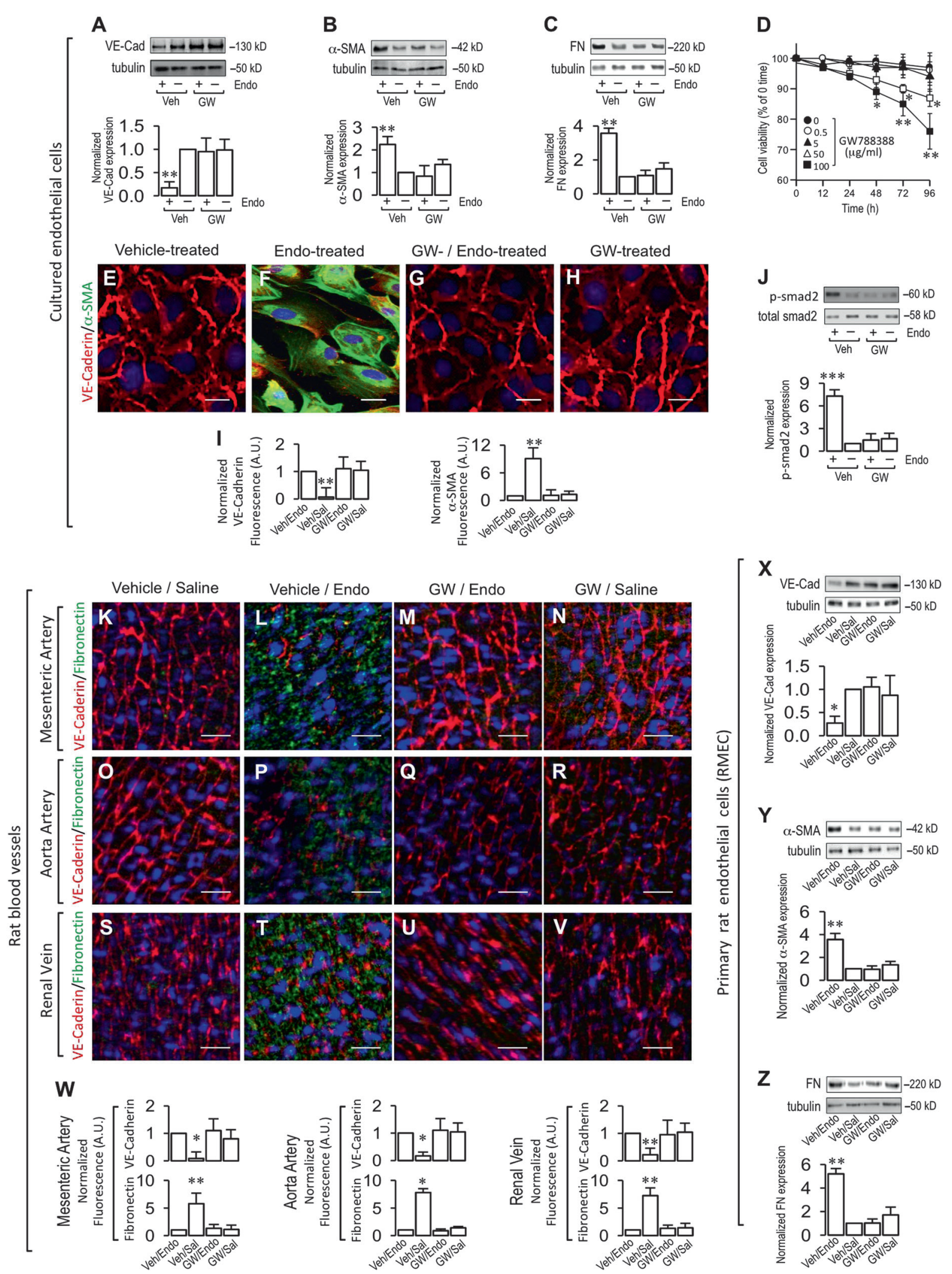

expression and an strongly increased FN expression (Fig. 11, p, t). These changes were concordant with endothelial fibrosis induced by endotoxemia. In contrast,
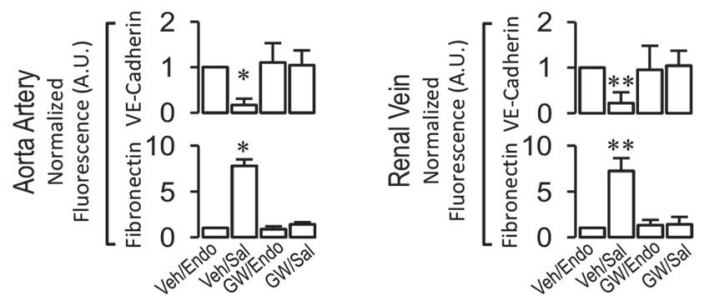

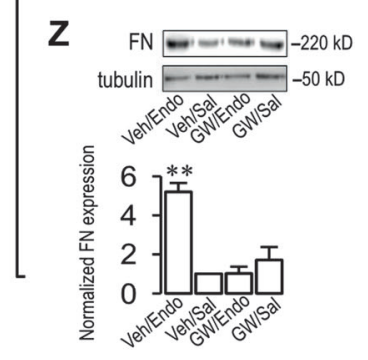

GW-788388-treated rats subjected to endotoxemia (GWtreated/endotoxemic group) were resistant to endotoxemia-induced endothelial fibrosis, showing 
Fig. 1 GW-788388 inhibits endothelial fibrosis both in vitro and in vivo at 24 and $72 \mathrm{~h}$, respectively. Representative images (upper panels) and densitometric analyses (lower panels) from western blot experiments performed for the detection of VE-Cadherin (a), $\alpha$-SMA (b), and fibronectin (c) in cultured endothelial cells treated with GW$788388(5 \mu \mathrm{g} / \mathrm{mL})$ in the presence or absence endotoxin $(20 \mu \mathrm{g} / \mathrm{mL})$ after $24 \mathrm{~h}$ of exposure. Protein levels were normalized against tubulin and expressed relative to vehicle-treated condition in the absence of endotoxin. Values are expressed as the mean $\pm \mathrm{SD}$. $* * p<0.05$, assessed by two-way ANOVA and the Bonferroni post-test $(N=4)$. d Cell viability assay in cultured endothelial cells treated with GW$788388(0,0.5,5,50$, and $100 \mu \mathrm{g} / \mathrm{mL})$ for $0,12,24,48,72,96 \mathrm{~h}$. Values are expressed as the mean \pm SD. $* p<0.05 ; * * p<0.01$. Every GW-788388 concentration was compared with the vehicle-treated condition at every time by two-way ANOVA and the Bonferroni posttest $(N=4)$. Representative images from fluorescence immunocytochemistry experiments performed for the detection of VE-Cadherin and $\alpha$-SMA in endothelial cells cultured in the following conditions: vehicle-treated (e), endotoxin-treated (f), GW-788388-treated/endotoxin-treated (g), and GW-788388-treated (h). Scale bar represents 40 $\mu \mathrm{m}$. $(N=4)$. i Fluorescence quantification of VE-Cadherin (left panel) and $\alpha$-SMA (right panel) was performed from experiments $(N=4)$ as showed in $\mathbf{e}-\mathbf{h}$. Values are expressed as the mean \pm SD. $* p<0.05 ; * * p$ $<0.01$, assessed by two-way ANOVA and the Bonferroni post-test. ( $N$ $=4$ ). Representative images (upper panels) and densitometric analyses (lower panels) from western blot experiments performed for the detection of p-smad-2 and total smad-2 in cultured endothelial cells treated with GW-788388 $(5 \mu \mathrm{g} / \mathrm{mL})$ in the presence or absence endotoxin $(20 \mu \mathrm{g} / \mathrm{mL})$ after $6 \mathrm{~h}$ of exposure $(\mathbf{j})$. p-smad-2 protein levels were normalized to those of total smad- 2 and expressed relative to those in the vehicle-treated condition in the absence of endotoxin. Values are expressed as the mean $\pm \mathrm{SD}$. $* * * p<0.001$, assessed by two-way ANOVA and the Bonferroni post-test $(N=3)$. Representative images from fluorescence immunohistochemistry experiments performed for the detection of VE-Cadherin and Fibronectin in mesenteric artery $(\mathbf{k}-\mathbf{n})$, aorta artery $(\mathbf{o}-\mathbf{r})$ and renal vein $(\mathbf{s}-\mathbf{v})$ extracted from rats subjected to the following conditions: vehicletreated/saline-treated (Vehicle/Saline) (k: mesenteric artery, o: aorta artery, s: renal vein), vehicle-treated/endotoxemic (Vehicle/ Endo) (l: mesenteric artery, p: aorta artery, t: renal vein), GW-788388treated/endotoxemic (GW/Endo) (m: mesenteric artery, $\mathbf{q}$ : aorta artery, u: renal vein), and GW-788388-treated/saline-treated (GW/Saline) (n: mesenteric artery, $\mathbf{r}$ : aorta artery, $\mathbf{v}$ : renal vein). Scale bar represents $50 \mu \mathrm{m}$. $(N=5)$. w Fluorescence quantification of VE-Cadherin (upper panels) and fibronectin (lower panels) was performed from several experiments as showed in mesenteric artery, aorta artery and renal vein. Values are expressed as the mean \pm SD. $* p<0.05 ; * * p<0.01$, assessed by two-way ANOVA and the Bonferroni post-test. $(N=5)$. Representative images (upper panels) and densitometric analyses (lower panels) from western blot experiments performed for the detection of VE-Cadherin (x), $\alpha$-SMA (y), and fibronectin $(\mathbf{z})$ in mesenteric primary endothelial cells (RMEC) extracted from rats subjected to the following conditions: vehicle-treated/saline-treated (Veh/Sal), vehicle-treated/endotoxemic (Veh/Endo), GW-788388treated/endotoxemic (GW/Endo), and GW-788388-treated/saline-treated (GW/Sal). GW-788388 was administered by gavage $(5 \mathrm{mg} / \mathrm{kg}$ a day) $24 \mathrm{~h}$ before and during endotoxemia. Endotoxemia was induced by the IP injection of LPS $(20 \mathrm{mg} / \mathrm{kg})$ for $72 \mathrm{~h}$. Values are expressed as the mean \pm SD. $* p<0.05 ; * * p<0.01$, assessed by two-way ANOVA and the Bonferroni post-test. $(N=3)$

normal VE-cadherin expression and no FN expression increase (Fig. 1m, q, u), similar to the observations in the vehicle-treated/endotoxemic group. GW-788388-treated rats subjected to saline solution IP injection (GW-treated/ saline-treated group) showed results similar to those in the vehicle-treated/saline-treated group (Fig 1n, r, v), indicating that GW-788388 treatment does not generate side effects.

Concordantly, fluorescence quantification of immunohistochemistry showed above indicated that VE-cadherin (Fig. 1w, upper panel) and FN (Fig. 1w, lower panel) fluorescence level were decreased and increased, respectively, compared with control conditions in mesenteric artery (Fig. 1w, left panel), aorta artery (Fig. 1w, middle panel), and renal vein (Fig. 1w, right panel) from endotoxemic rats (vehicle-treated/endotoxemic group). However, blood vessels extracted from GW-788388-treated rats subjected to endotoxemia (GW-treated/endotoxemic group) failed to change VE-cadherin and FN fluorescence level (Fig. 1w).

In addition to this, fluorescent immunohistochemistry experiments were performed to determine the capacity of GW-788388 treatment to inhibit smad-2 phosphorylation as a measure of ALK5 activity inhibition. Vehicle-treated/ saline-treated and GW-treated/saline-treated groups showed almost not detectable p-smad-2 staining. Vehicle-treated/ endotoxemic group showed increased p-smad-2 labeling, however, GW-treated/endotoxemic group showed decreased p-smad-2 staining (Supplementary Figure S1AD), denoting that GW-788388 is effective to inhibit ALK5 activity in our in vivo study model.

In addition, primary mesenteric endothelial cells (RMEC) were extracted from rats of all the experimental groups mentioned above to study the ability of GW-788388 to inhibit the protein expression change associated with endotoxemia-induced endothelial fibrosis. The vehicletreated/endotoxemic condition showed a significant decrease in the expression of the endothelial marker VEcadherin and increased expression of both the fibrotic protein $\alpha-S M A$ and $\mathrm{FN}$ (Fig. 1x-z, respectively), compared with the vehicle-treated/saline-treated and GW-treated/saline-treated group conditions. However, the GW-treated/ endotoxemic group failed to exhibit both decreased VEcadherin levels (Fig. 1x) and increased levels of the fibrotic proteins $\alpha$-SMA (Fig. 1y) and FN (Fig. 1z). Furthermore, RMECs were extracted from rats of all the experimental groups mentioned above to determine the capacity of GW788388 treatment to inhibit smad-2 phosphorylation as a measure of ALK5 activity inhibition. Our results indicated that vehicle-treated/saline-treated and GW-treated/salinetreated groups showed almost not detectable p-smad-2 levels. As we expected, vehicle-treated/endotoxemic group showed increased p-smad-2 level. In contrast, GW-treated/ endotoxemic group did not show p-smad-2 increased level (Supplementary Figure S1E, F), indicating that GW-788388 is effective to inhibit ALK5 activity in our in vivo study model. 
All these results show that GW-788388 treatment is effective at inhibiting in vivo endotoxemia-induced endothelial fibrosis.

To demonstrate that endotoxin administration to rats is effective at inducing cardiovascular changes concordant with the accepted criteria for the diagnosis of severe sepsis in humans, which is characterized by hypotension, tachycardia, and tachypnea [41, 42], we recorded the $\mathrm{P}_{\mathrm{S}}, \mathrm{f}_{\mathrm{H}}$, tidal volume $\left(V_{T}\right)$, respiratory frequency $\left(f_{R}\right)$, and minute ventilatory volume $\left(\mathrm{V}_{\mathrm{E}}: \mathrm{V}_{\mathrm{T}} \times \mathrm{f}_{\mathrm{R}}\right)$ in rats IP injected with saline solution or endotoxin after $24 \mathrm{~h}$ of treatment. The rats subjected to endotoxemia showed decreased systolic blood pressure (hypotension), tachycardia, and tachypnea compared with saline-treated rats, indicating that the treatment produced hemodynamic alterations concordant with severe sepsis (Supplementary Table S3).

Once our in vivo models of both GW-788388 treatment to inhibit endotoxemia-induced endothelial fibrosis and endotoxemia induction in rats were established, we proceeded to investigate the effects of endothelial fibrosis inhibition on $P_{S}$ and $f_{H}$ during endotoxemia. To that end, we measured variables after endotoxemia induction for up to $72 \mathrm{~h}$.

Rats were treated with the GW-788388 vehicle solution and subjected to sham endotoxemia (vehicle-treated/salinetreated group) $24 \mathrm{~h}$ later. These animals did not show changes in $P_{S}$ and $f_{H}$ compared with the GW-788388treated rats that were injected with saline solution (GWtreated/saline-treated group) (Fig. 2a, b, respectively). However, vehicle-treated rats subjected to endotoxemia (vehicle-treated/endotoxemic group) showed significantly decreased $P_{S}$ values (Fig. 2a) and increased $f_{H}$ values (Fig. 2b) at $24 \mathrm{~h}$ after endotoxemia challenge. These changes were concordant with the induction of endotoxemia. Notably, the GW-788388-treated rats subjected to endotoxemia (GW-treated/endotoxemic group) were resistant to changes in $P_{S}$ and $f_{H}$ levels, showing values equal to those in vehicle-treated/saline-treated rats (Fig. 2a, b, respectively). These results suggest that the inhibition of endotoxemia-induced endothelial fibrosis by GW-788388 treatment generates a protective action to maintain normal $P_{S}$ and $f_{H}$ levels during endotoxemia.

\section{Endotoxemia-induced endothelial fibrosis inhibition protects endotoxemic rats against MODS and hypoglycemia}

MODS is a major morbidity factor underlying severe sepsis and affects the function of several organs, including the liver, kidneys, and muscles. Furthermore, metabolic function is altered, producing severe hypoglycemia after $12 \mathrm{~h}$ of sepsis induction. To investigate whether the inhibition of endothelial fibrosis during endotoxemia is beneficial for
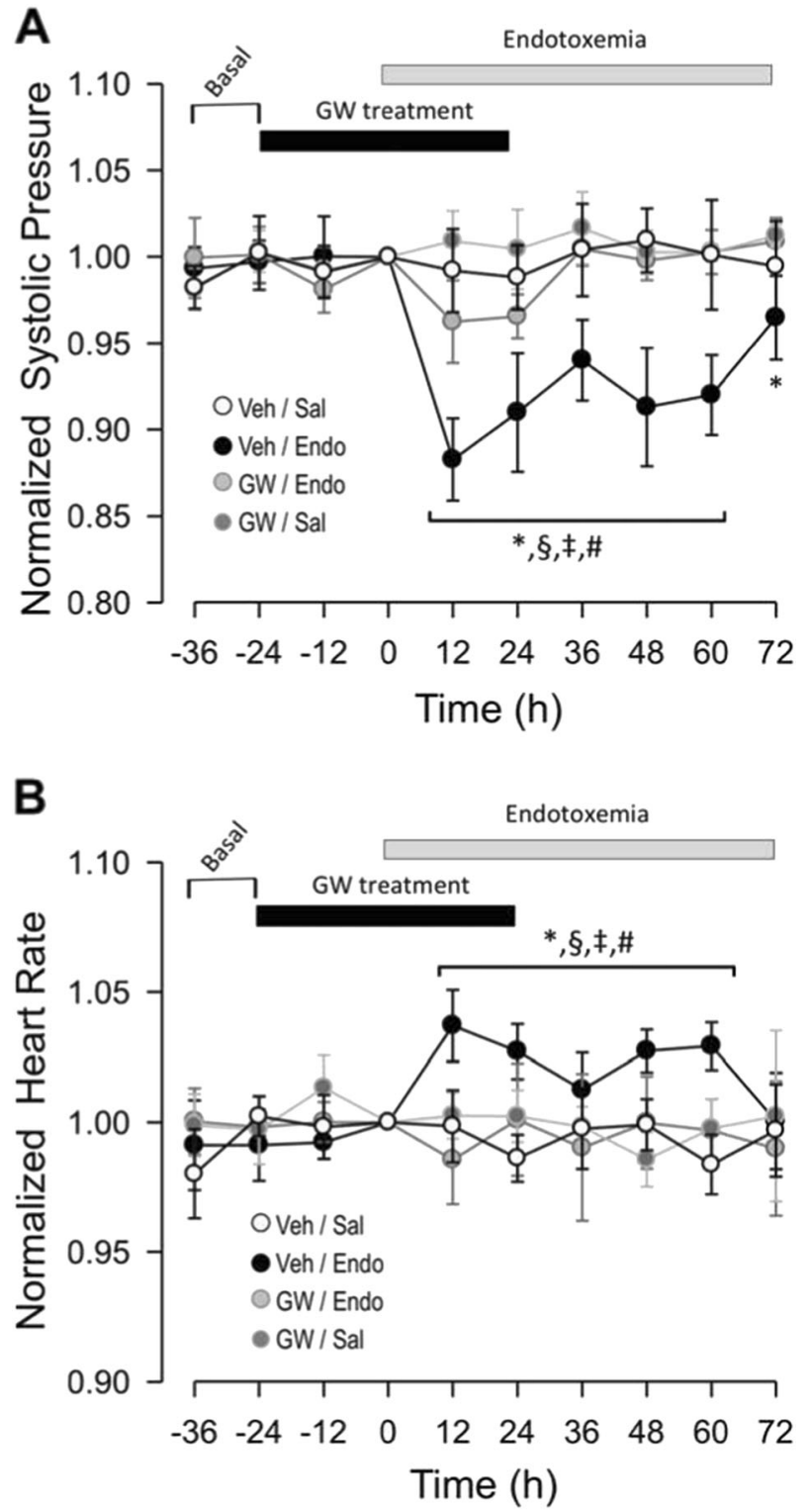

Fig. 2 GW-induced endothelial fibrosis inhibition improves hypotension and tachycardia in 72-h endotoxemic rats. Systolic blood pressure $\left(\mathrm{P}_{\mathrm{S}}\right)$ (a) and heart rate $\left(\mathrm{f}_{\mathrm{H}}\right)($ b) variables in vehicle-treated/salinetreated rats (open circles, $N=8$ ), GW-treated/saline-treated rats (dark gray circles, $N=8$ ), vehicle-treated/endotoxemic rats (closed circles, $N=8$ ), and GW-treated/endotoxemic rats (gray circles, $N=8$ ). GW788388 was administrated by gavage $(5 \mathrm{mg} / \mathrm{kg}$ a day) $24 \mathrm{~h}$ before and during 72-h endotoxemia. After 1 day of GW-788388 administration, endotoxemia was produced by IP injection of $20 \mathrm{mg} / \mathrm{kg}$ LPS. Animals were recorded $12 \mathrm{~h}$ in basal condition (basal) and then $24 \mathrm{~h}$ with GW788388 or vehicle administration (GW treatment). Next, animals were challenged with LPS or saline solution for $72 \mathrm{~h}$. Values are expressed as the mean $\pm \mathrm{SD}$. $* p<0.05$, vehicle-treated/endotoxemic group compared with basal, assessed by ANOVA and Dunnett's post-test. ${ }^{\S} p$ $<0.05$, vehicle-treated/endotoxemic group compared with GW-treated/ endotoxemic group, assessed by two-way ANOVA and the Bonferroni post-test. ${ }^{\ddagger} p<0.05$, vehicle-treated/endotoxemic group compared with vehicle-treated/saline-treated group, assessed by two-way ANOVA and the Bonferroni post-test. ${ }^{\#} p<0.05$, vehicle-treated/endotoxemic group compared with $\mathrm{GW}$-treated/saline-treated group, assessed by two-way ANOVA and the Bonferroni post-test. $N=8$ 

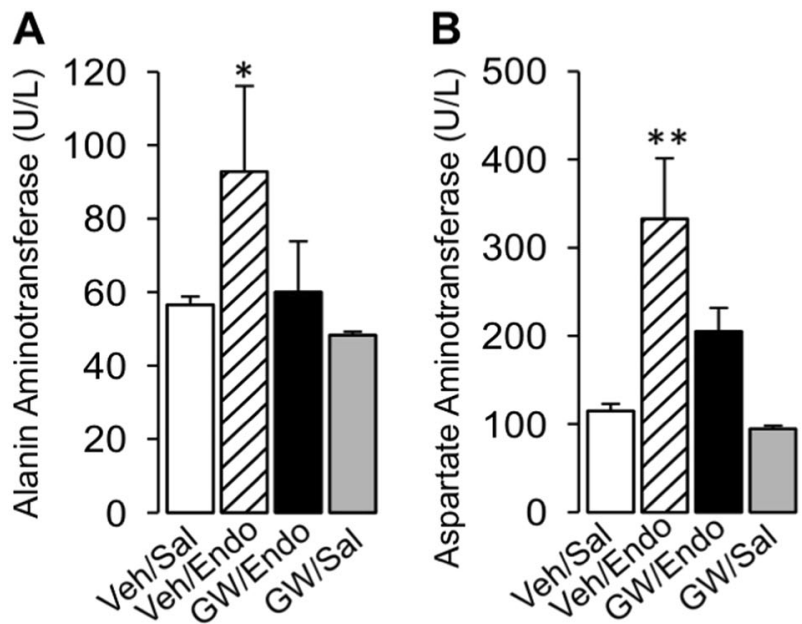

Fig. 3 GW-induced endothelial fibrosis inhibition improves MODS in 24-h endotoxemic rats. Alanine aminotransferase (a), aspartate aminotransferase (b), total bilirubin (c), and gamma-glutamyl transferase (d) for the liver function and blood urea nitrogen (e) and creatinine (f) for the kidney function were measured in vehicle-treated/saline-treated rats (open bars, $N=8$ ), vehicle-treated/endotoxemic rats (dashed bars, $N=8$ ), GW-treated/endotoxemic rats (closed bars, $N=8$ ), and GWtreated/saline-treated rats (dark gray bars, $N=8$ ). GW-788388 was administrated by gavage $(5 \mathrm{mg} / \mathrm{kg}$ a day) $24 \mathrm{~h}$ before and during $24-\mathrm{h}$ endotoxemia. Values are expressed as the mean \pm SD. $* p<0.05 ; * * p$ $<0.01$, assessed by one-way ANOVA (Kruskal-Wallis) and Dunn's post-test. $N=8$

C
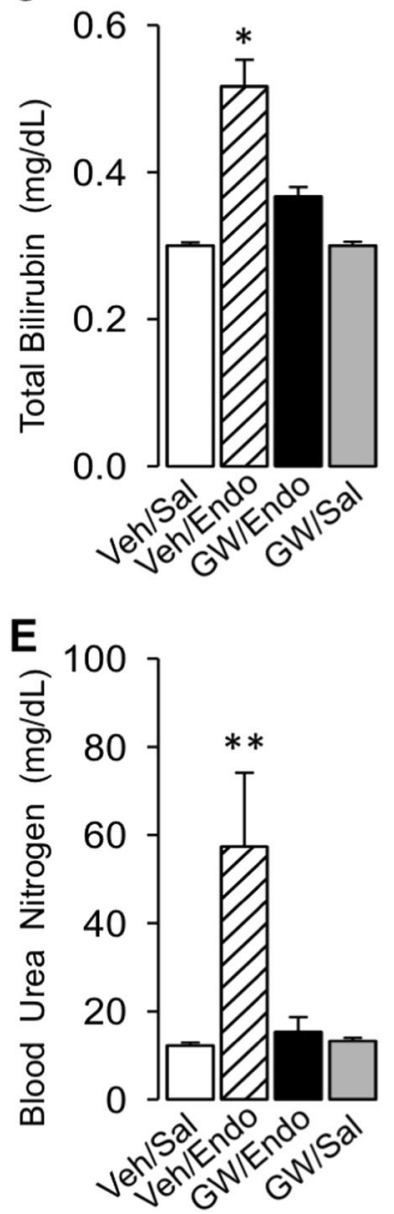

D

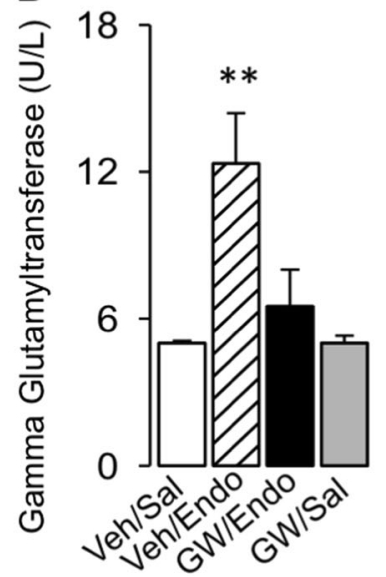

$\mathbf{F}$

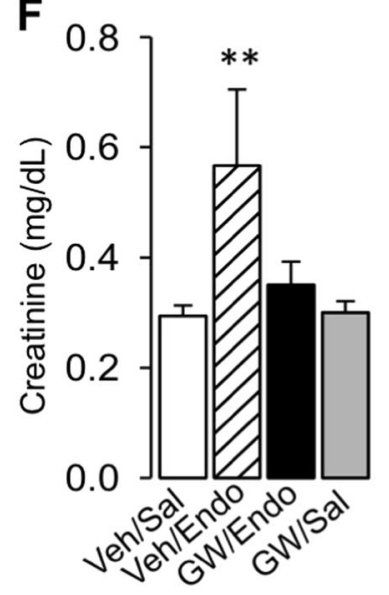

preserving normal liver and kidney function during endotoxemia, we measured the serum markers of liver and kidney dysfunction in GW-788388-treated endotoxemic rats after $24 \mathrm{~h}$ of endotoxemia induction.

The vehicle-treated/saline-treated and GW-treated/salinetreated rats showed no significant differences in their plasma levels; the levels of the liver dysfunction markers

ALT, AST, TBIL, or GGT; or in the levels of kidney dysfunction markers CRE and BUN (Fig. 3a-f). Salinetreated/endotoxemic rats show increased ALT, AST, TBIL, GGT, CRE, and BUN levels, denoting endotoxemiainduced dysfunction generation in the liver and kidney (Fig. 3a-f). Notably, the GW-treated/endotoxemic rats showed normal levels of liver and kidney marker dysfunction, showing similar values for ALT, AST, TBIL, GGT, $\mathrm{CRE}$, and BUN compared with those in the vehicle-treated/ saline-treated and GW-treated/saline-treated groups (Fig. 3a-f).

Histopathological examination performed by hematoxylin and eosin staining corroborated that GW treatment had positive effects on liver and kidney function during endotoxemia. After endotoxemia induction, liver tissue sections showed morphological changes, apparition of cytoplasmic vacuoles, erythrocyte accumulation, and polymorphonuclear infiltration (Fig. 4b, b') compared with those in the control condition (Fig. 4a). Interestingly, endotoxemiainduced signs of damage were decreased in GW-treated animals (Fig. 4c, c'), as indicated by a blindly scored grading scale for liver damage (Fig. 4e). Similarly, kidney samples showed enhanced renal cell swelling, glomerular structure alteration and swelling, vacuolar degeneration and tubular cell necrosis (Fig. 4g, g'). These observed detrimental features of renal endotoxemia-induced damage were decreased when endothelial fibrosis inhibition was induced (Fig. 4h, h'), as indicated by a blindly scored grading scale for kidney damage (Fig. $4 \mathrm{j}$ ).

To assess the impact of inhibiting endotoxemia-induced endothelial fibrosis in liver and kidney fibrogenesis during endotoxemia, liver and kidney sections were stained with picroSirius red to detect collagen depositions. Liver tissue from endotoxemic rats exhibited high fibrosis, showing marked collagen fiber deposition and loss of hepatic architecture (Fig. 5b, b') compared with tissues in the control condition (Fig. 5a). Hepatic fibrosis induced by endotoxemia was severely decreased in GW-treated animals (Fig. 5c, c'), showing significant $\mathrm{GW}$-induced protection (Fig. 5e). Similarly, endotoxemia-induced kidney fibrosis (Fig. $5 \mathrm{~g}, \mathrm{~g}^{\prime}$ ) was significantly decreased when rats were 

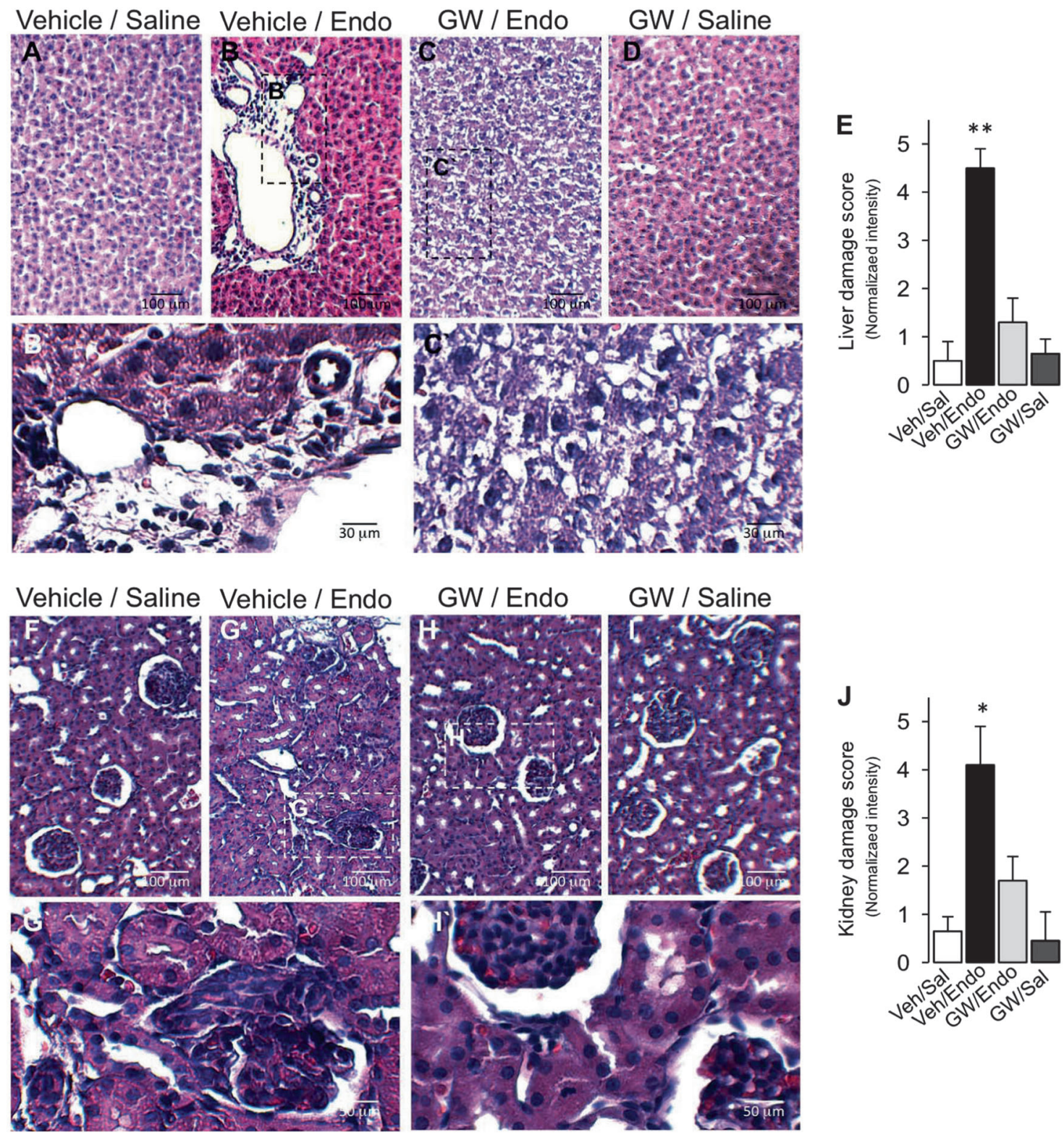

Fig. $4 \mathrm{GW}$-induced endothelial fibrosis inhibition decreases liver and kidney tissue damage in 24-h endotoxemic rats. Representative images of hematoxylin/eosin-stained liver (a-d) and kidney (f-i) sections from vehicle-treated/saline-treated rats $(\mathbf{a}, \mathbf{f})$, vehicle-treated/endotoxemic rats $(\mathbf{b}, \mathbf{g})$, GW-treated/endotoxemic rats $(\mathbf{c}, \mathbf{h})$, and $\mathrm{GW}$ treated/saline-treated rats $(\mathbf{d}, \mathbf{i})$, after $24 \mathrm{~h}$. GW-788388 was administrated by gavage $(5 \mathrm{mg} / \mathrm{kg}$ a day) $24 \mathrm{~h}$ before and during $24-\mathrm{h}$ endotoxemia. Images were subjected to blind analysis of tissue liver (e) and kidney (j) damage using the following grading scale for tissue damage: normal $=0$, minimal $=1$, mild $=2$, moderate $=3$, marked $=$ 4 , and severe $=5$. Values are expressed as the mean \pm SD. ${ }^{*} p<0.05$, $* * p<0.01$, assessed by one-way ANOVA (Kruskal-Wallis) and Dunn's post-test. $N=8$

study the expression changes of the fibrosis markers collagen III (col III) and FN and to determine the effect of GW788388. Representative portions of liver and kidney were extracted and subjected to analysis immediately after treatment.

Our results indicated that liver and kidney samples from vehicle-treated/saline-treated and GW-treated/saline-treated groups showed similar levels Col III and FN (Fig. 51-s, for liver and kidney, respectively). In contrast, vehicle-treated/ were extracted from rats of all the experimental groups to 

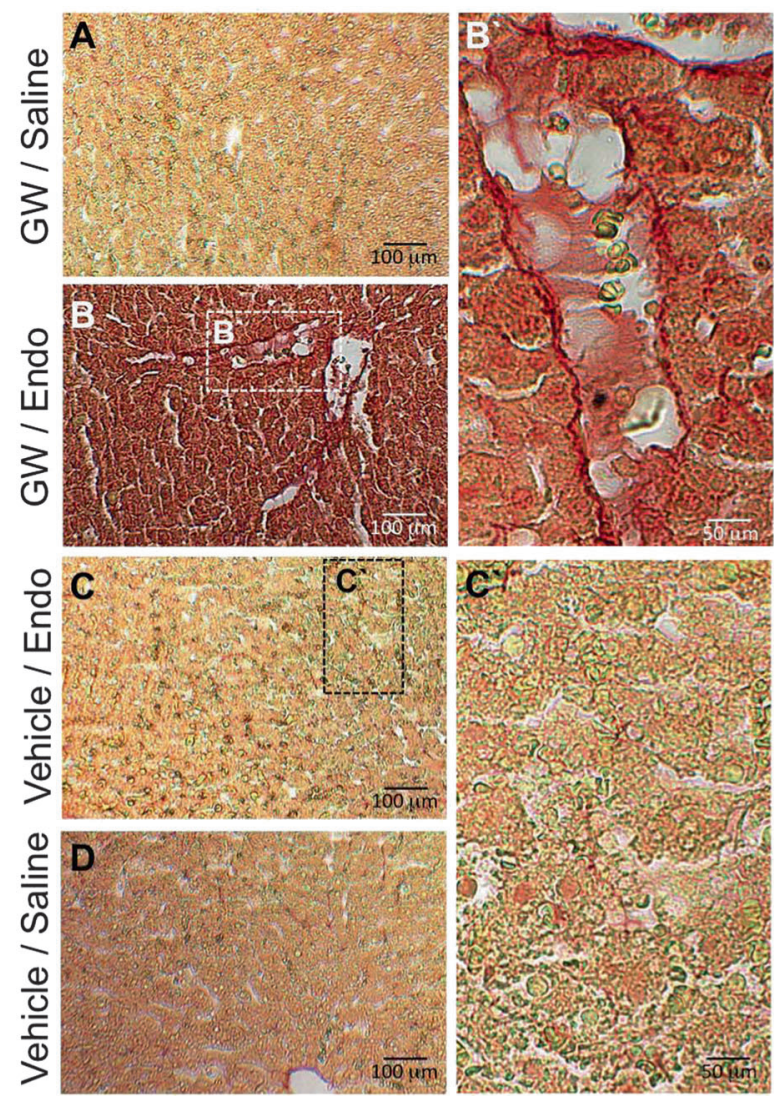

E

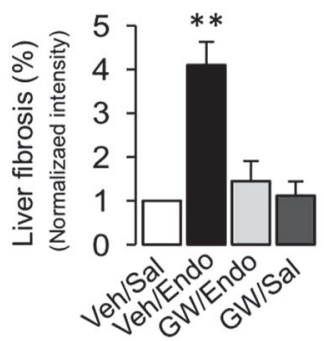

$\mathbf{L}$
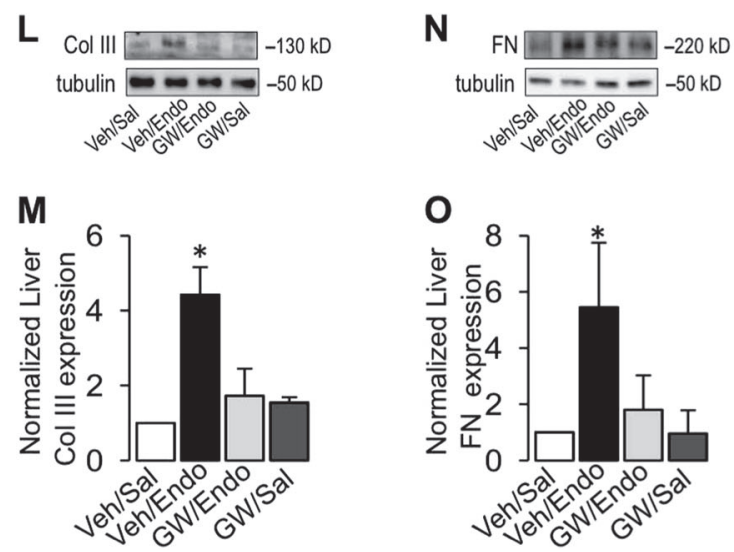

endotoxemic group showed increased levels of col III and FN. Interestingly, GW-treated endotoxic animals (GWtreated/endotoxemic group) were resistant to col III and FN
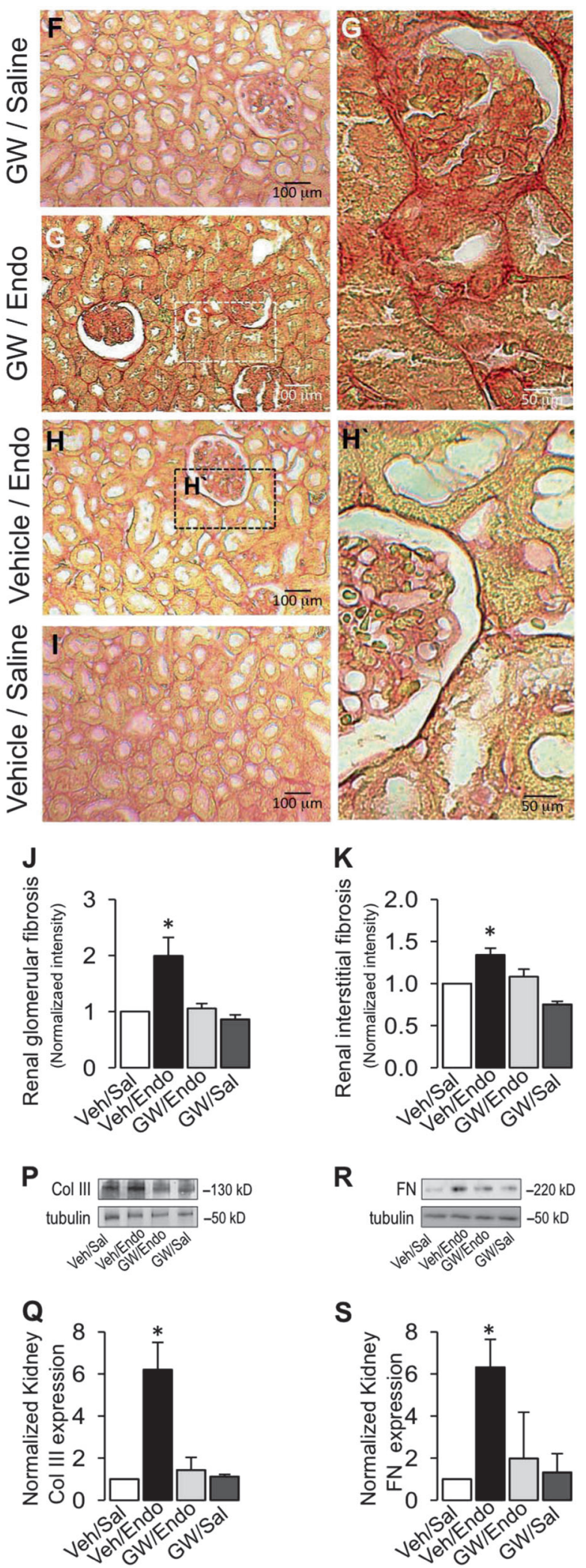

increase (Fig. 51-s, for liver and kidney, respectively). These results showed that GW-788388 is effective to inhibit the endotoxemia-induced col III and FN increase, 
Fig. 5 GW-induced endothelial fibrosis inhibition decreases liver and kidney tissue fibrosis in 24-h endotoxemic rats. Representative images of picroSirius red stained liver (a-d) and kidney (f-l) sections from vehicle-treated/saline-treated rats $(\mathbf{a}, \mathbf{f})$, vehicle-treated/endotoxemic rats $(\mathbf{b}, \mathbf{g}), \mathrm{GW}$-treated/endotoxemic rats $(\mathbf{c}, \mathbf{h})$ and $\mathrm{GW}$-treated/salinetreated rats $(\mathbf{d}, \mathbf{i})$, after $24 \mathrm{~h}$. GW-788388 was administrated by gavage $(5 \mathrm{mg} / \mathrm{kg}$ a day) $24 \mathrm{~h}$ before and during $24-\mathrm{h}$ endotoxemia. Images were subjected to analysis of tissue fibrosis in liver (e) and kidney (glomerular, $\mathbf{j}$ and interstitial, $\mathbf{k})$. Values are expressed as the mean \pm SD. ${ }^{*} p<0.05, * * p<0.01$, assessed by one-way ANOVA (KruskalWallis) and Dunn's post-test. $N=8$. Representative images $(\mathbf{l}, \mathbf{n}, \mathbf{p}, \mathbf{r})$ and densitometric analyses $(\mathbf{m}, \mathbf{o}, \mathbf{q}, \mathbf{s})$ from western blot experiments performed for the detection of collagen III $(\mathbf{l}, \mathbf{m}, \mathbf{p}, \mathbf{q})$ and fibronectin $(\mathbf{n}, \mathbf{o}, \mathbf{r}, \mathbf{s})$ protein levels in liver $(\mathbf{l}, \mathbf{m}, \mathbf{n}, \mathbf{o})$ and kidney $(\mathbf{p}, \mathbf{q}, \mathbf{r}, \mathbf{s})$. Values are expressed as the mean \pm SD. ${ }^{* *} p<0.01$, assessed by oneway ANOVA (Kruskal-Wallis) and Dunn's post-test. $N=6$
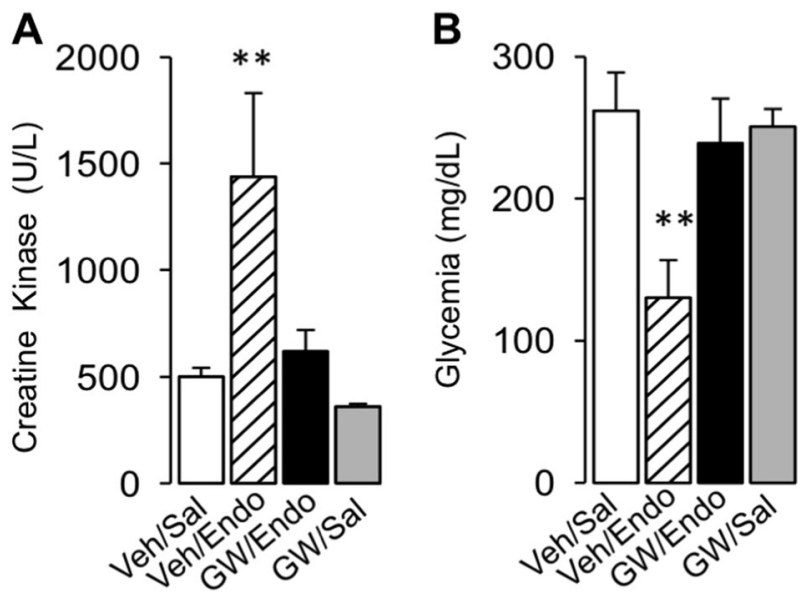

Fig. $6 \mathrm{GW}$-induced endothelial fibrosis inhibition improves mass muscle wasting and hypoglycemia in 24-h endotoxemic rats. Creatine kinase (a) for muscle mass wasting and glycemia (b) for glycemia control were measured in vehicle-treated/saline-treated rats (open bars, $N=8$ ), vehicle-treated/endotoxemic rats (dashed bars, $N=8$ ), $\mathrm{GW}$ treated/endotoxemic rats (closed bars, $N=8$ ), and GW-treated/salinetreated rats (dark gray bars, $N=8$ ). GW-788388 was administrated by gavage $(5 \mathrm{mg} / \mathrm{kg}$ a day) $24 \mathrm{~h}$ before and during $24-\mathrm{h}$ endotoxemia. Values are expressed as the mean $\pm \mathrm{SD}$. ${ }^{*} p<0.01$, assessed by oneway ANOVA (Kruskal-Wallis) and Dunn's post-test. $N=8$

suggesting that GW-788388 treatment is able to inhibit the endotoxemia-induced fibrosis.

Muscle mass is severely diminished during severe sepsis and endotoxemia via cachexia. Therefore, we evaluated whether GW-788388 treatment protected against muscle mass wasting during endotoxemia. The vehicle-treated/saline-treated and GW-treated/saline-treated rats showed no changes in their plasma levels of CK (Fig. 6a), whereas vehicle-treated/endotoxemic rats showed increased $\mathrm{CK}$ serum levels (Fig. 6a), indicating cachexia. However, GWtreated/endotoxemic rats exhibited $\mathrm{CK}$ levels similar to those observed in the vehicle-treated/saline-treated and GW-treated/saline-treated groups (Fig. 6a), indicating that
GW-788388 treatment protects endotoxemic rats against muscle mass wasting.

Hypoglycemia occurs in severe sepsis. Thus, we investigated whether GW-788388 treatment improves endotoxemia-induced hypoglycemia. The vehicle-treated/ saline-treated and GW-treated/saline-treated rats showed no changes in their GLY levels (Fig. 6b). In contrast, severe hypoglycemia was observed in the vehicle-treated/endotoxemic rats group, which was in agreement with previously published data [43-45] (Fig. 6b). Remarkably, the GWtreated/endotoxemic rats showed GLY levels similar to those in the vehicle-treated/saline-treated and GW-treated/ saline-treated groups (Fig. 6b), indicating that GW-788388 treatment maintains normal blood glucose levels during endotoxemia.

These results indicate that GW-788388 treatment protects against MODS, avoiding liver and kidney dysfunction, muscle mass wasting, and hypoglycemia during endotoxemia.

\section{Endotoxemia-induced endothelial fibrosis inhibition decreases endothelial dysfunction protein expression}

When the endothelium is exposed to endotoxin, it enters in an activated state [2-4]. Endothelial activation leads to an increase in the expression of adhesion molecules, such as vWF, which promotes binding of platelets or leukocytes and TF, which is the initiator of the extrinsic coagulation pathway [10-21]. To determine whether endotoxemiainduced endothelial fibrosis inhibition mediated by GW788388 modulates activation of endothelial cells, TF and vWF were measured in RMEC extracted from treated animals. Our results indicated that vehicle-treated/saline-treated and GW-treated/saline-treated groups showed similar mRNA (Fig. 7a, b) and protein (Fig. 7c-f) levels of TF and vWF. When, rats were subjected to endotoxemia (vehicletreated/endotoxemic group), mRNA and protein levels of $\mathrm{TF}$ and vWF were strongly increased (Fig. 7). In contrast, GW-treated/endotoxemic group did not show increased mRNA and protein levels of TF and vWF (Fig. 7). These results showed that $\mathrm{GW}-788388$ is effective to inhibit the endotoxemia-induced TF and vWF increase expression at mRNA and protein level, suggesting that GW-788388 inhibits the endothelial activation induced by endotoxemia.

\section{Endotoxemia-induced endothelial fibrosis inhibition prevents increased pro-inflammatory cytokine expression and oxidative stress}

As endotoxemia is characterized by an uncontrolled inflammatory response, we investigated whether GW- 


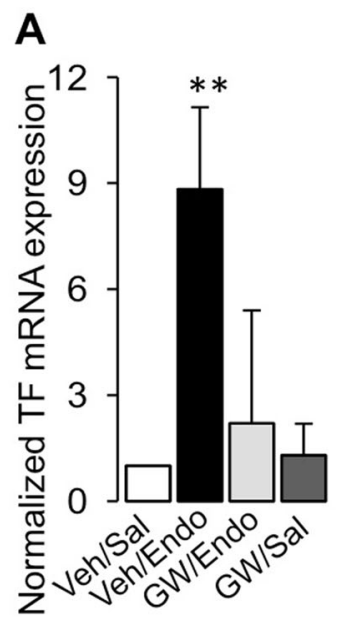

B
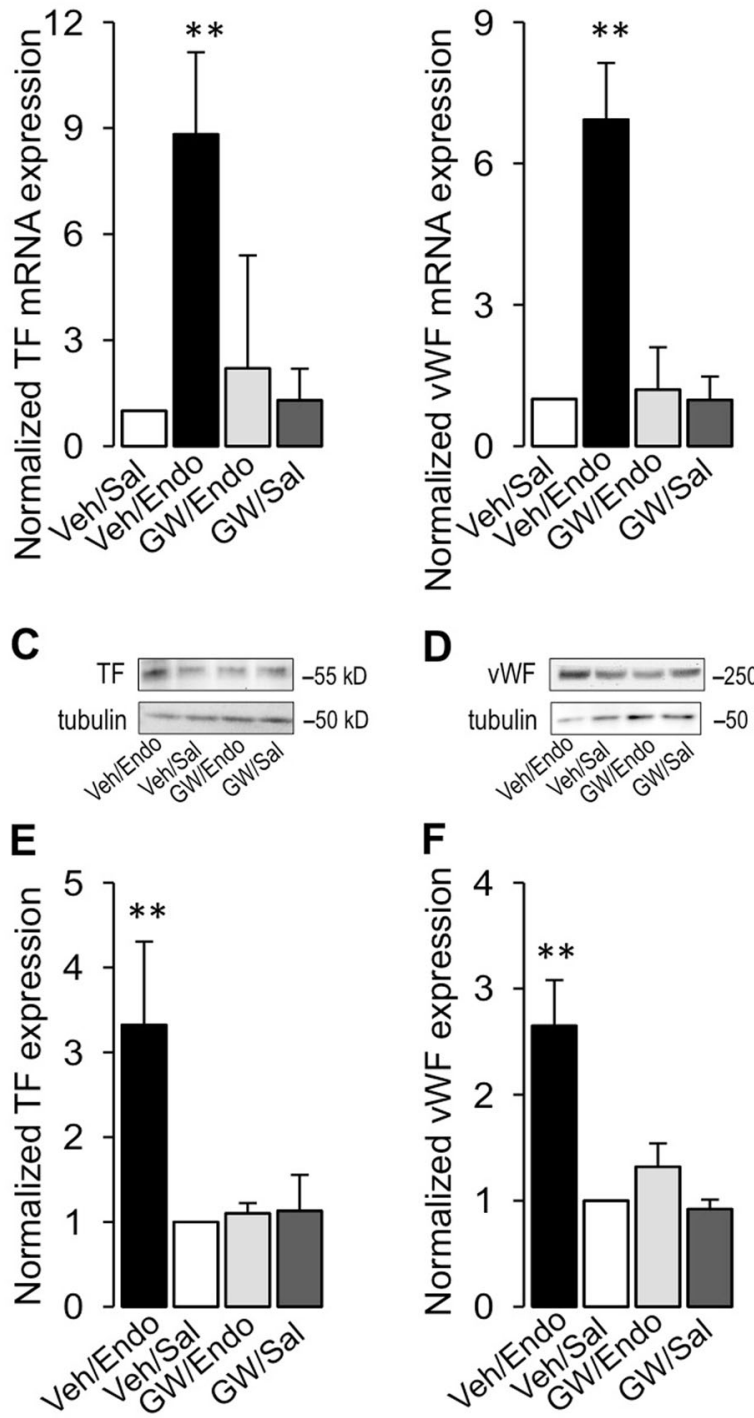

levels compared with those in the vehicle-treated/salinetreated rats at 12, 24, 48, and $72 \mathrm{~h}$ (Fig. 8a-f, respectively). However, vehicle-treated/endotoxemic rats showed strongly increased TNF- $\alpha$, IL-1 $\beta$, IL- 6 , and IL-12 pro-inflammatory cytokines measured at 12 and $24 \mathrm{~h}$, denoting a severe inflammatory response evoked by endotoxemia (Fig. 8a-d, respectively). For TNF- $\alpha$, a minor but still significant increase was observed at $72 \mathrm{~h}$. In addition to this, the levels of the anti-inflammatory cytokines IL-4 and IL-10 did not change at 12,24 , and $48 \mathrm{~h}$, but their levels were increased at $72 \mathrm{~h}$ after endotoxin challenge. (Fig. 8e, f, respectively). Interestingly, the GW-treated rats subjected to endotoxemia failed to exhibit changes in pro- and anti-inflammatory cytokine levels at any time point measured, and instead showed values that did not differ from those in vehicletreated/saline-treated and $\mathrm{GW}$-treated/saline-treated rats. (Fig. 8a-f, respectively),

Furthermore, GW-788388 treatment was able to decreased oxidative stress generation during endotoxemia. GW-treated/saline-treated rats showed no changes in their blood ROS levels compared with those in vehicle-treated/ saline-treated rats as measured using the ROS-sensitive dye dichlorodihydrofluorescein (DCF) (Fig. 9a) and diacetate DHE, a probe mostly selective to detect superoxide anions (Fig. 9b). Concordant with the oxidative burst observed during sepsis, vehicle-treated/endotoxemic rats showed strongly increased ROS levels, indicating that endotoxemia generates a severe oxidative stress environment (Fig. 9a, b, respectively). However, the GW-treated/endotoxemic rats were resistant to ROS increases (Fig. 9a, b, respectively), showing values equal to those in the vehicle-treated/salinetreated and GW-treated/saline-treated rats. In addition, the endogenous antioxidant agents reduced GSH and $\alpha$ tocopherol were also modulated by GW-788388 treatment. GW-treated/saline-treated rats showed no differences in their GSH and $\alpha$-tocopherol blood levels compared with those in the vehicle-treated/saline-treated rats (Fig. 9c, d, respectively), whereas the vehicle-treated/endotoxemic rats showed decreased GSH and $\alpha$-tocopherol levels, indicating that endotoxemia generates a lessened antioxidant condition. However, GW-treated/endotoxemic rats showed GSH and $\alpha$-tocopherol levels similar to those in the vehicletreated/saline-treated and $\mathrm{GW}$-treated/saline-treated rats (Fig. 9c, d, respectively).

Considering that $\mathrm{NAD}(\mathrm{P}) \mathrm{H}$ oxidase is an important source of intracellular ROS, we tested the participation of the endothelial isoforms of NOX; NOX-1, NOX-2, and NOX-4 [4, 46, 47]. To that end, RMECs were extracted from rats of all the experimental groups, to study the mRNA expression changes of NOX-1, NOX-2, and NOX-4. RMECs were extracted and subjected to RT-qPCR experiments immediately after treatment. Our results indicated that GW-treated/saline-treated groups showed similar levels 


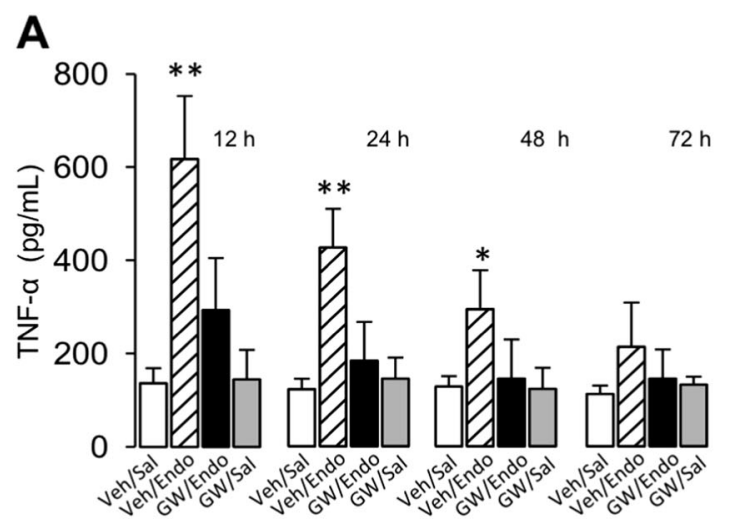

B
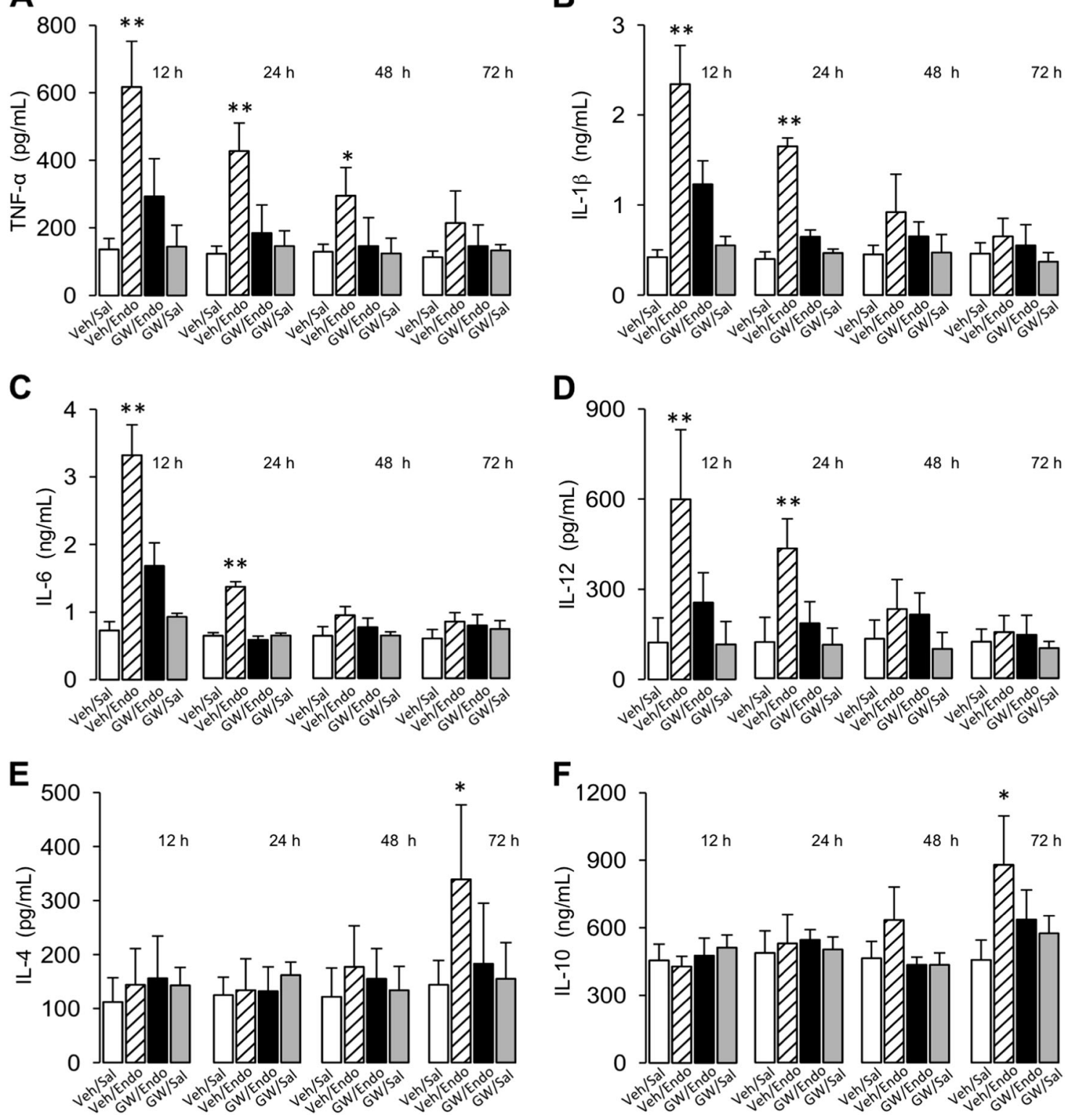

Fig. $8 \mathrm{GW}$-induced endothelial fibrosis inhibition decreases proinflammatory cytokines secretion in 72 -h endotoxemic rats. Plasma levels of TNF- $\alpha(\mathbf{a})$, IL-1 $\beta$ (b), IL-6 (c), IL-12 (d), IL-4 (e), and IL-10 (f) were measured at 12, 24, 48, $72 \mathrm{~h}$, in vehicle-treated/saline-treated rats (open bars, $N=8$ ), vehicle-treated/endotoxemic rats (dashed bars, $N=8$ ), GW-treated/endotoxemic rats (closed bars, $N=8$ ), and

of NOX-1, NOX-2, and NOX-4 mRNA levels compared with vehicle-treated/saline-treated group (Fig. 9e-g). In contrast, vehicle-treated/endotoxemic group showed increased mRNA levels of NOX-2 (Fig. 9f), whereas NOX1 and NOX-4 did not show any change (Fig. 9e, g). Interestingly, GW-treated/endotoxemic group did not show increased levels of NOX-2 (Fig. 9e-g).

These results showed that GW-788388 is effective to inhibit the endotoxemia-induced NOX-2 mRNA expression increase, suggesting that GW-788388 inhibits the
$\mathrm{GW}$-treated/saline-treated rats (dark gray bars, $N=8$ ). GW-788388 was administrated by gavage $(5 \mathrm{mg} / \mathrm{kg}$ a day) $24 \mathrm{~h}$ before and during 72-h endotoxemia. Values are expressed as the mean \pm SD. $* p<$ $0.05, * * p<0.01$, assessed by one-way ANOVA (Kruskal-Wallis) and Dunn's post-test

endotoxemia-induced oxidative stress burst by the inhibition of the specific NOX-2 isoforms.

\section{Endotoxemia-induced endothelial fibrosis inhibition decreases the relative risk of death during endotoxemia}

Concordant with the high mortality observed with sepsis syndrome, contingency analysis performed using a Fisher's exact test showed that GW-788388 treatment decreased the 
Fig. $9 \mathrm{GW}$-induced endothelial fibrosis inhibition decreases oxidative burst in 24-h endotoxemic rats. Plasma levels of oxidative stress measured as DCF (a) and DHE (b)

fluorescence, reduced glutathione content (c), and $\alpha$ tocopherol content (d) were measured in vehicle-treated/ saline-treated rats (open bars, $N=8$ ), vehicle-treated/ endotoxemic rats (dashed bars, $N=8), \mathrm{GW}$-treated/ endotoxemic rats (closed bars, $N=8$ ), and GW-treated/salinetreated rats (dark gray bars, $N=$ 8). GW-788388 was administrated by gavage ( $5 \mathrm{mg} / \mathrm{kg}$ a day) $24 \mathrm{~h}$ before and during 24-h endotoxemia. Values are expressed as the mean \pm SD. $* p<0.05 ; * * p<$ 0.01 , assessed by one-way ANOVA (Kruskal-Wallis) and Dunn's post-test. Endotoxemiainduced endothelial NOX isoform mRNA expression (NOX-1 (e), NOX-2 (f), and NOX-4 (g)) was determined in vehicle-treated/saline-treated rats (open bars, $N=5$ ), vehicletreated/endotoxemic rats (dashed bars, $N=5$ ), GW-treated/ endotoxemic rats (closed bars, $N=5$ ), and GW-treated/salinetreated rats (dark gray bars, $N=$ 5). GW-788388 was administrated by gavage $(5 \mathrm{mg} / \mathrm{kg}$ a day) $24 \mathrm{~h}$ before and during 24-h endotoxemia. Values are expressed as the mean \pm SD. $* p<0.05$, assessed by one-way ANOVA (KruskalWallis) and Dunn's post-test
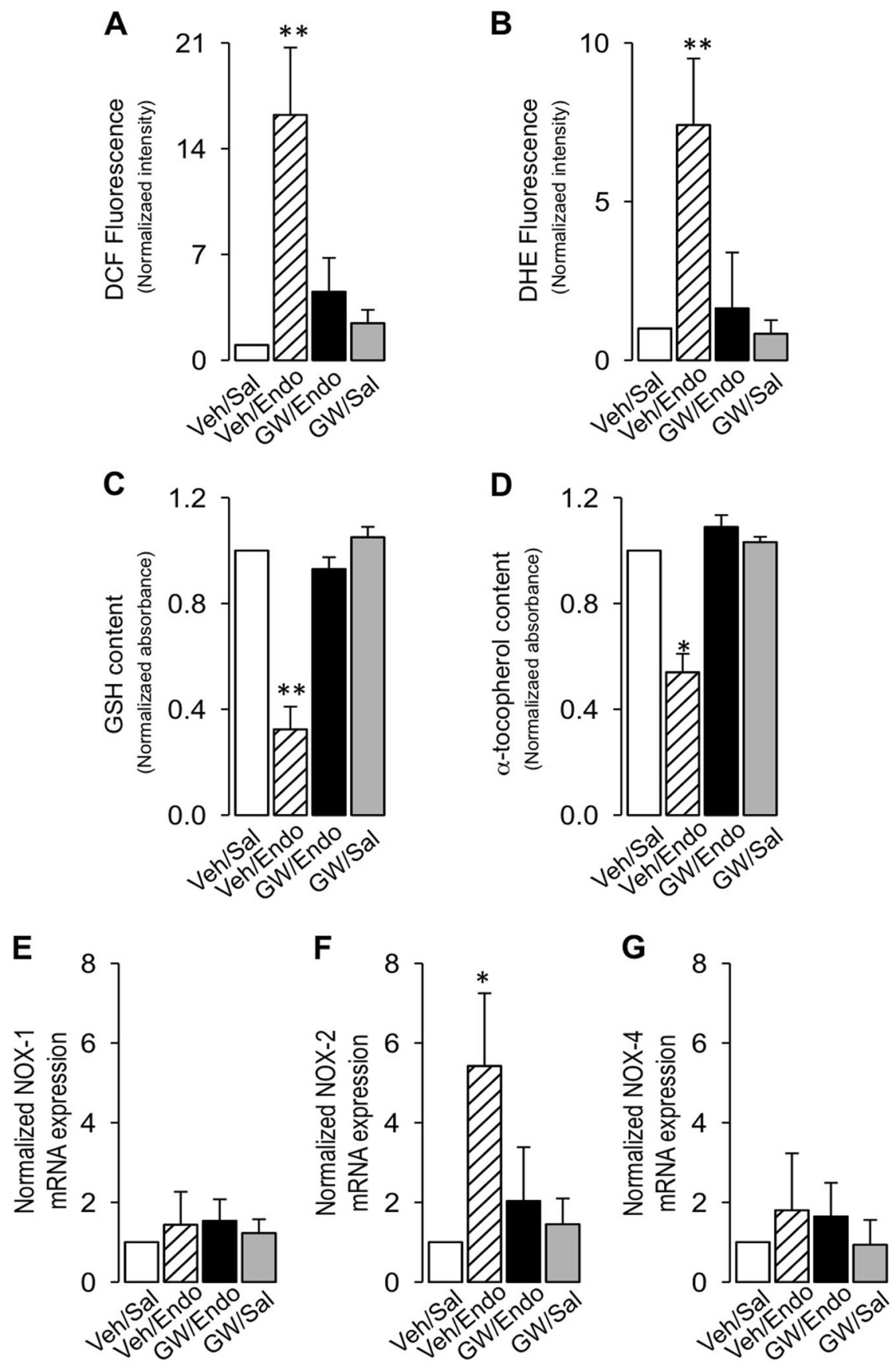

risk of death in endotoxemic rats. Endotoxemic rats (vehicle-treated/endotoxemic) showed an elevated risk of death compared with those in the vehicle-treated/saline-treated and GW-treated/saline-treated groups after $72 \mathrm{~h}$ of endotoxemia induction. Notably, endotoxemic rats treated with GW-788388 (GW-treated/endotoxemic) showed a decreased risk of death, exhibiting significant differences from the vehicle-treated/endotoxemic rats, whereas no differences were observed when compared with the vehicletreated/saline-treated and $\mathrm{GW}$-treated/saline-treated rats after $72 \mathrm{~h}$ of endotoxemia (Fig. 10a).
The risk of death was significantly decreased in the GWtreated/endotoxemic group when curves were analyzed using a log-rank test (also called Mantel-Cox test). A comparison of survival curves from the vehicle-treated/saline-treated and $\mathrm{GW}$-treated/saline-treated rats showed no differences between groups. By contrast, significant differences were observed when these survival curves were compared with the curve from endotoxemic rats (vehicle-treated/endotoxemic rats). Remarkably, survival curve analysis of the GW-treated/ endotoxemic group revealed that despite the deaths recorded, GW-788388 treatment to endotoxemic animals decreased 

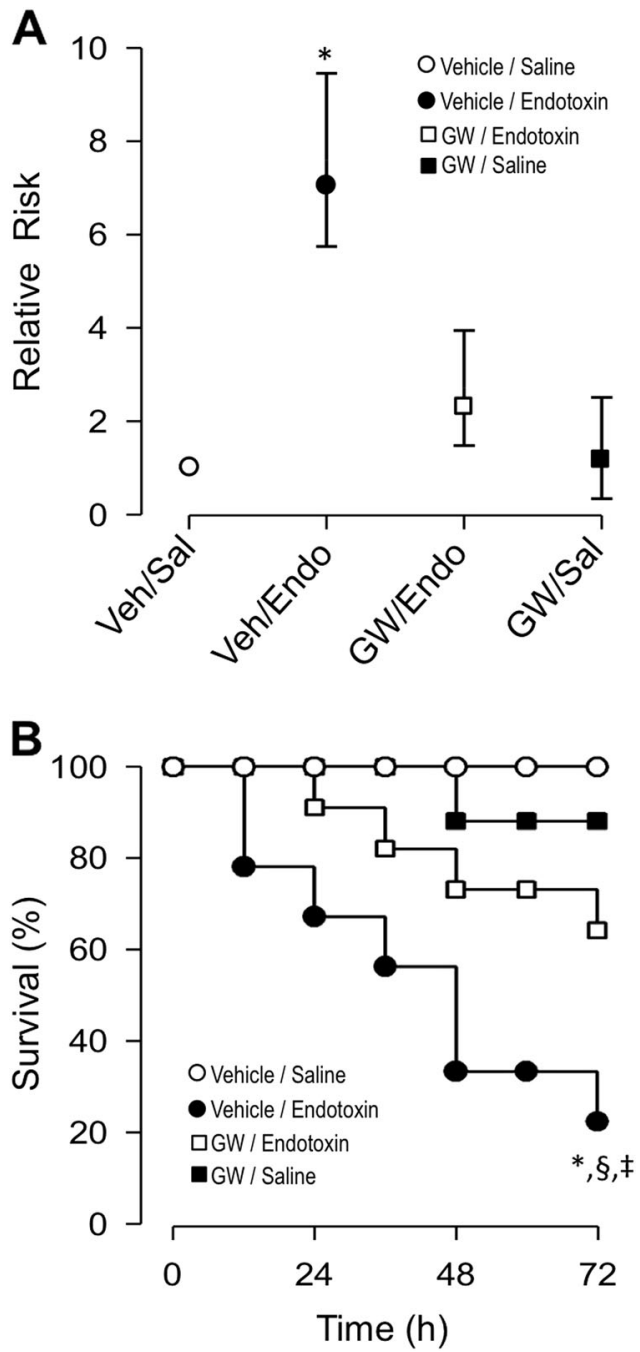

Fig. $10 \mathrm{GW}$-induced endothelial fibrosis inhibition decreases the risk of death and mortality in 72-h endotoxemic rats. Contingency analyses (a) and survival (Kaplan-Meier) curves (b) determined in vehicletreated/saline-treated rats (open circles, $N=8$ ), GW-treated/salinetreated rats (closed squares, $N=8$ ), vehicle-treated/endotoxemic rats (closed circles, $N=8$ ), and GW-treated/endotoxemic rats (open squares, $N=8$ ), after $72 \mathrm{~h}$. In (a), values are expressed as the mean \pm $95 \%$ confidence interval (CI). ${ }^{*} p=0.0122$ (Fisher's exact test) comparing vehicle-treated/endotoxemic rats versus GW-treated/endotoxemic group. $p=0.0028$ when comparing vehicle-treated/ endotoxemic rats versus vehicle-treated/saline-treated group. $p=$ 0.0066 when comparing vehicle-treated/endotoxemic rats versus $\mathrm{GW}$ treated/saline-treated group. In (b), ${ }^{*} p=0.0016,{ }^{\circledR} p=0.0063,{ }^{\ddagger} p=$ 0.0275 (log-rank (Mantel-Cox) test) when comparing vehicle-treated/ endotoxemic rats compared versus vehicle-treated/saline-treated, GWtreated/saline-treated and GW-treated/endotoxemic rats, respectively

their risk of death. A comparison of the survival curve from GW-treated/endotoxemic group revealed that this condition was not different from those in the vehicle-treated/salinetreated and GW-treated/saline-treated groups, whereas a comparison versus the vehicle-treated/endotoxemic condition showed a significant difference, indicating that the relative risk of death decreased. Giving more weight to deaths at early time points at the time of analysis (Gehan-Breslow-Wilcoxon test) showed that GW-788388 treatment also decreased the risk of death in this early time frame. A comparison of the GW-treated/endotoxemic group survival curves showed that neither the vehicle-treated/saline-treated groups nor the GWtreated/saline-treated groups differed from the GW-treated/ endotoxemic group, whereas the vehicle-treated/endotoxemic group showed an increased risk of death (Fig. 10b).

\section{Discussion}

It is known that endotoxic conditions generate a fibrotic protein reprogramming in endothelial cells, thus altering endothelial function; however, whether endotoxemiainduced endothelial fibrosis is responsible for endotoxemia pathogenesis and mortality remains unanswered. The results reported herein suggest that the inhibition of endotoxemia-induced endothelial fibrosis exerts protective actions against endotoxemia by maintaining blood pressure to preserve normal perfusion and lessening both the immune response and oxidative stress.

Our results show for the first time that the inhibition of endotoxemia-induced endothelial fibrosis is protective against endotoxemia. GW-788388 treatment is efficient at reducing both the hypotension and tachycardia observed during endotoxemia, maintaining these parameters to near physiologically normal levels. In addition, MODS induced by endotoxemia is decreased by GW-788388 treatment, showing normal functions of the liver and kidney, muscle mass, and GLY levels. In concordance, the liver and kidney showed normal histology and reduced signs of fibrosis when endotoxemia-induced endothelial fibrosis was inhibited in endotoxemic animals. Furthermore, pro-inflammatory cytokine production, oxidative stress and antioxidant molecules decreased when endotoxic animals were treated with GW-788388. Notably, endotoxemia-induced endothelial fibrosis resulted in significantly increased survival against sepsis, decreasing the risk of death associated with endotoxemia at early and late time points.

Ten years ago, endothelial cells exposed to TGF- $\beta$ were originally reported to undergo a conversion to fibroblasts via a mechanism mediated by the endothelial-tomesenchymal transition (EndMT) process [28, 48]. This seminal finding of the initial evidence showing EndMT in adult tissue was confirmed by several others groups reporting that EndMT was induced by several inflammatory agents, such as TNF- $\alpha$, IL-6, and IL-1 $\beta$ [27-32]. During the last 5 years, we reported that the conversion of endothelial cells to activated fibroblasts by EndMT is also induced by the bacterial LPS, opening a wide debate regarding the roles of this conversion in the initiation and progression of inflammatory diseases [25, 26, 33]. From this point of view, 
the most severe infectious inflammatory disease is sepsis, which is called endotoxemia when caused by an endotoxin from a Gram-negative bacterial infection. It is well known that vascular endothelial cells exposed to endotoxin undergo extensive cell death $[4,49,50]$. However, a significant portion of endotoxin-exposed endothelial cells escape from death and exhibit a conversion to fibroblasts [26]. Fibrosis progression is possibly the mechanism used by endothelial cells to acquire resistance to endotoxininduced death. However, as a consequence, at least three deleterious processes are possibly activated: (i) endothelial cells converted to fibroblasts acquire impaired function, (ii) fibrosed endothelial cells alter the normal function of the intact endothelium, and (iii) fibrosed endothelial cells avoid endothelial turnover by progenitor endothelial cells.

The control of hypotension during sepsis is of primary importance. For that reason, the results shown herein demonstrating that the inhibition of endotoxemia-induced endothelial fibrosis by GW-788388 treatment can improve hypotension and tachycardia during endotoxemia are of major relevance. Because hypotension is a key feature of sepsis syndrome, a usual approach to sepsis treatment is the administration of vasoconstrictors and inotropic agents to achieve adequate blood pressure. Unfortunately, in several cases, this strategy frequently fails to revert low blood pressure to normal levels [10-16]. An important consequence of hypotension and especially of refractory hypotension is the insufficient blood perfusion to organs. Normal blood pressure guarantees sufficient organ perfusion to ensure a suitable provision of nutrients and waste clearance in accordance with organ requirements [18, 19]. In septic patients, signs of organ hypoperfusion are frequently observed, most likely due to a diminished blood pressure, which severely alters organ function and increases the effects of MODS [19, 23, 24].

During sepsis, there is an increased production of the vasodilator agent $\mathrm{NO}$ as a consequence of inducible $\mathrm{NO}$ synthase expression in response to endotoxin and proinflammatory cytokines, which generates increased levels of NO acting on smooth muscle cells of the microvasculature [51, 52]. In the same line, the loss of sympathetic action in septic patients [53] is reported, which suggests a deficient adrenergic tone to elicit vasoconstriction. In fact, $\beta$ adrenergic inhibition is beneficial for sepsis treatment [54, 55]. However, whether endothelial fibrosis modifies NO production or the $\beta$-adrenergic sympathetic tone during sepsis to improve vascular reactivity is unknown.

Another factor that induces organ damage during sepsis is immune system overactivation, which implies increased proinflammatory cytokine secretion and oxidative stress burst. Pro-inflammatory cytokines, such as TNF- $\alpha$, extensively damage parenchymatous organs, which subsequently causes MODS [56-58]. Our results showed that GW-treated endotoxemic animals were resistant to the incremental plasma concentrations of TNF- $\alpha$, IL-1 $\beta$, IL- 6 , and IL-12. In light of this, we expected to observe reduced levels of organ damage biochemical markers in GW-treated septic animals [58, 59]. In fact, GW treatment decreased MODS in endotoxemic rats, correlating with the decreased plasma concentration of cytokines. In addition, oxidative stress is well known as a key agent generating MODS during sepsis [60, 61]. In concordance, antioxidants are used to protect against MODS during sepsis [62-64]. GW treatment is efficient at decreasing plasma oxidative stress and increasing diminished levels of endogenous antioxidants. GLY changes during sepsis [41, 42]. Sepsis shows an early hyperglycemic phase followed by a late hypoglycemic phase. A hyperglycemic phase is observed in the first $2 \mathrm{~h}$ of sepsis, whereas after $24 \mathrm{~h}$, a severe hypoglycemic phase [43-45] is observed. In concordance, GLY measurements performed at $24 \mathrm{~h}$ after endotoxemia induction showed a hypoglycemic stage. Remarkably, GWtreated endotoxemic animals showed normal GLY and were resistant to hypoglycemia.

Inhibition of endotoxemia-induced endothelial fibrosis increases the survival rate of endotoxemic rats. This protective action is based on reversing the decreases in blood pressure associated with MODS, and inhibition of oxidative bursts and pro-inflammatory cytokine secretion. Notably, the risk of death was significantly decreased in endotoxemic animals treated with GW-788388. Since sepsis syndrome is the main cause of death in critically ill patients, and the therapy currently employed is unsatisfactory, the evidence presented herein represents a step forward in preventing sepsis pathogenesis and highlights a novel target that may be used to generate therapeutic alternatives that lower the mortality of septic patients.

Acknowledgements This work was supported by research grants from Fondo Nacional de Desarrollo Científico y Tecnológico-FONDECYT 1161288 (FS), 11170840 (CE), and 1161646 (CC-V), Millennium Institute on Immunology and Immunotherapy P09-016-F (FS, CC-V), UNAB DI-741-15/N (FS, CC-V).

\section{Compliance with ethical standards}

Conflict of interest The authors declare that they have no conflict of interest.

Publisher's note: Springer Nature remains neutral with regard to jurisdictional claims in published maps and institutional affiliations.

\section{References}

1. Zarbock A, Gomez H, Kellum JA. Sepsis-induced acute kidney injury revisited: pathophysiology, prevention and future therapies. Curr Opin Crit Care. 2014;20:588-95.

2. Riedemann NC, Guo R-F, Ward PA. The enigma of sepsis. J Clin Invest. 2003;112:460-7. 
3. Pinsky MR. Dysregulation of the immune response in severe sepsis. Am J Med Sci. 2004;328:220-9.

4. Simon F, Fernández R. Early lipopolysaccharide-induced reactive oxygen species production evokes necrotic cell death in human umbilical vein endothelial cells. J Hypertens. 2009;27:1202-16.

5. Karima R, Matsumoto S, Higashi H, Matsushima K. The molecular pathogenesis of endotoxic shock and organ failure. Mol Med Today. 1999;5:123-32.

6. Rivers EP, Ahrens T. Improving outcomes for severe sepsis and septic shock: tools for early identification of at-risk patients and treatment protocol implementation. Crit Care Clin. 2008;24(3 Suppl):S1-47.

7. Winters BD, Eberlein M, Leung J, Needham DM, Provonost PJ, Sevransky JE. Long-term mortality and quality of life in sepsis: a systematic review. Crit Care Med. 2010;38:1276-83.

8. Angus DC, Linde-Zwirble WT, Lidicker J, Clermont G, Carcillo J, Pinsky MR. Epidemiology of severe sepsis in the United States: analysis of incidence, outcome, and associated costs of care. Crit Care Med. 2001;29:1303-10.

9. Brun-Buisson C, Meshaka P, Pinton P, Vallet B. EPISEPSIS: a reappraisal of the epidemiology and outcome of severe sepsis in French intensive care units. Intensive Care Med. 2004;30:580-8.

10. Vallet B. Bench-to-bedside review: endothelial cell dysfunction in severe sepsis: a role in organ dysfunction? Crit Care. 2003;7:130-8.

11. De Backer D, Creteur J, Preiser J-C, Dubois MJ, Vincent JL. Microvascular blood flow is altered in patients with sepsis. Am J Respir Crit Care Med. 2002;166:98-104.

12. Bateman RM, Sharpe MD, Ellis CG. Bench-to-bedside review: microvascular dysfunction in sepsis--hemodynamics, oxygen transport, and nitric oxide. Crit Care. 2003;7:359-73.

13. Sakr Y, Dubois M-J, De Backer D, Creteur J, Vincent JL. Persistent microcirculatory alterations are associated with organ failure and death in patients with septic shock. Crit Care Med. 2004;32:1825-31.

14. Trzeciak S, Dellinger RP, Parrillo JE, Guglielmi M, Bajaj J, Abate $\mathrm{NL}$, et al. Early microcirculatory perfusion derangements in patients with severe sepsis and septic shock: relationship to hemodynamics, oxygen transport, and survival. Ann Emerg Med. 2007;49:88-98.

15. Dellinger RP, Levy MM, Rhodes A, Annane D, Gerlach H, Opal SM, et al. Surviving sepsis campaign: International Guidelines for Management of Severe Sepsis and Septic Shock, 2012. Intensive Care Med. 2013;39:165-228.

16. Hollenberg SM, Ahrens TS, Annane D, Astiz ME, Chalfin DB, Dasta JF, et al. Practice parameters for hemodynamic support of sepsis in adult patients: 2004 update. Crit Care Med. 2004;32:1928-48.

17. De Backer D, Donadello K, Sakr Y, Ospina-Tascon G, Salgado D, Scolleta $S$, et al. Microcirculatory alterations in patients with severe sepsis: impact of time of assessment and relationship with outcome. Crit Care Med. 2013;41:791-9.

18. Marshall JC. Inflammation, coagulopathy, and the pathogenesis of multiple organ dysfunction syndrome. Crit Care Med. 2001;29(7 Suppl):S99-106.

19. Ziesmann MT, Marshall JC. Multiple organ dysfunction: the defining syndrome of sepsis. Surg Infect. 2018;19:184-90.

20. Spicer A, Calfee CS. Fixing the leak: targeting the vascular endothelium in sepsis. Crit Care. 2012;16:177.

21. Curry FRE, Adamson RH. Vascular permeability modulation at the cell, microvessel, or whole organ level: towards closing gaps in our knowledge. Cardiovasc Res. 2010;87:218-29.

22. Vincent J-L, Taccone F, Schmit X. Classification, incidence, and outcomes of sepsis and multiple organ failure. Contrib Nephrol. 2007;156:64-74.
23. Englert JA, Fink MP. The multiple organ dysfunction syndrome and late-phase mortality in sepsis. Curr Infect Dis Rep. 2005;7:335-41.

24. Baue AE. Sepsis, systemic inflammatory response syndrome, multiple organ dysfunction syndrome, and multiple organ failure: are trauma surgeons lumpers or splitters? J Trauma. 2003;55:997-8.

25. Echeverría C, Montorfano I, Tapia P, Riedel C, Cabello-Verrugio C, Simon F. Endotoxin-induced endothelial fibrosis is dependent on expression of transforming growth factors $\beta 1$ and $\beta 2$. Infect Immun. 2014;82:3678-86.

26. Echeverría C, Montorfano I, Sarmiento D, Becerra A, NuñezVillena F, Figueroa XF, et al. Lipopolysaccharide induces a fibrotic-like phenotype in endothelial cells. J Cell Mol Med. 2013;17:800-14.

27. Sarmiento D, Montorfano I, Cáceres M, Echeverría C, Fernández $\mathrm{R}$, Cabello-Verrugio C, et al. Endotoxin-induced vascular endothelial cell migration is dependent on TLR4/NF- $\mathrm{KB}$ pathway, $\mathrm{NAD}(\mathrm{P}) \mathrm{H}$ oxidase activation, and transient receptor potential melastatin 7 calcium channel activity. Int J Biochem Cell Biol. 2014;55:11-23.

28. Zeisberg EM, Potenta S, Xie L, Zeisberg M, Kalluri R. Discovery of endothelial to mesenchymal transition as a source for carcinoma-associated fibroblasts. Cancer Res. 2007;67:10123-8.

29. Maleszewska M, Moonen J-RAJ, Huijkman N, van de Sluis B, Krenning G, Harmsen MC. IL- $1 \beta$ and TGF $\beta 2$ synergistically induce endothelial to mesenchymal transition in an NFkBdependent manner. Immunobiology. 2013;218:443-54.

30. Mahler GJ, Farrar EJ, Butcher JT. Inflammatory cytokines promote mesenchymal transformation in embryonic and adult valve endothelial cells. Arterioscler Thromb Vasc Biol. 2012;33:121-30.

31. Potenta S, Zeisberg E, Kalluri R. The role of endothelial-tomesenchymal transition in cancer progression. $\mathrm{Br} \mathrm{J}$ Cancer. 2008;99:1375-9.

32. Montorfano I, Becerra A, Cerro R, Echeverría C, Sáez E, Fernandez $\mathrm{R}$, et al. Oxidative stress mediates the conversion of endothelial cells into myofibroblasts via a TGF- $\beta 1$ and TGF- $\beta 2-$ dependent pathway. Lab Invest. 2014;94:1068-82.

33. Pérez L, Muñoz-Durango N, Riedel CA, Echeverría C, Kalergis AM, Cabello-Verrugio C, et al. Endothelial-to-mesenchymal transition: cytokine-mediated pathways that determine endothelial fibrosis under inflammatory conditions. Cytokine Growth Factor Rev. 2017;33:41-54.

34. Echeverría C, Montorfano I, Cabello-Verrugio C, Armisén R, Varela D, Simon F. Suppression of transient receptor potential melastatin 4 expression promotes conversion of endothelial cells into fibroblasts via transforming growth factor/activin receptorlike kinase 5 pathway. J Hypertens. 2015;33:981-92.

35. Becerra A, Rojas M, Vallejos A, Villegas V, Pérez L, CabelloVerrugio $\mathrm{C}$, et al. Endothelial fibrosis induced by suppressed STAT3 expression mediated by signaling involving the TGF- $\beta 1 /$ ALK5/Smad pathway. Lab Invest. 2017;97:1033-46.

36. Gellibert F, de Gouville A-C, Woolven J, Mathews N, Nguyen VL, Bertho-Ruault C, et al. Discovery of 4-\{4-[3-(Pyridin-2-yl)-1 H-pyrazol-4-yl]pyridin-2-yl - $N$-(tetrahydro-2 H-pyran-4-yl)benzamide (GW788388): a potent, selective, and orally active transforming growth factor- $\beta$ type I receptor inhibitor. J Med Chem. 2006;49:2210-21.

37. de Oliveira FL, Araújo-Jorge TC, de Souza EM, de Oliveira GM, Degrave WM, Feige JJ, et al. Oral administration of GW788388, an inhibitor of transforming growth factor beta signaling, prevents heart fibrosis in chagas disease. PLoS Negl Trop Dis. 2012;6: e1696-14.

38. Edgell CJ, McDonald CC, Graham JB. Permanent cell line expressing human factor VIII-related antigen established by hybridization. Proc Natl Acad Sci USA. 1983;80:3734-7. 
39. Petersen M, Thorikay M, Deckers M, van Dinther M, Grygielko ET, Gellibert F, et al. Oral administration of GW788388, an inhibitor of TGF-beta type I and II receptor kinases, decreases renal fibrosis. Kidney Int. 2008;73:705-15.

40. Micha D, Voermans E, Eekhoff MEW, van Essen HW, ZandiehDoulabi B, Netelenbos C, et al. Inhibition of TGF $\beta$ signaling decreases osteogenic differentiation of fibrodysplasia ossificans progressiva fibroblasts in a novel in vitro model of the disease. Bone. 2016;84:169-80.

41. Levy MM, Fink MP, Marshall JC, Abraham E, Angus D, Cook D, et al. 2001 SCCM/ESICM/ACCP/ATS/SIS International Sepsis Definitions Conference. Crit Care Med. 2003;31:1250-6.

42. Levy MM, Fink MP, Marshall JC, Abraham E, Angus D, Cook D, et al. $2001 \mathrm{SCCM} / \mathrm{ESICM} / \mathrm{ACCP} / \mathrm{ATS} / \mathrm{SIS}$ International Sepsis Definitions Conference. Intensive Care Med. 2003;29:530-8.

43. Maitra SR, Wojnar MM, Lang CH. Alterations in tissue glucose uptake during the hyperglycemic and hypoglycemic phases of sepsis. Shock. 2000;13:379-85.

44. Igaki N, Matsuda T, Hirota Y, Kawaguchi T, Tamada F, Goto T. Streptococcal toxic shock syndrome presenting with spontaneous hypoglycemia in a chronic hemodialysis patient: pathophysiological mechanisms. Intern Med. 2003;42:421-3.

45. Tsai S-H, Lin Y-Y, Hsu C-W, Cheng CS, Chu DM. Hypoglycemia revisited in the acute care setting. Yonsei Med J. 2011;52:898-908.

46. Hwang J, Kleinhenz DJ, Lassègue B, Griendling KK, Dikalov S, Hart CM. Peroxisome proliferator-activated receptor-gamma ligands regulate endothelial membrane superoxide production. Am J Physiol Cell Physiol. 2005;288:C899-905.

47. Simon F, Stutzin A. Protein kinase C-mediated phosphorylation of p47phox modulates platelet-derived growth factor-induced $\mathrm{H} 2 \mathrm{O} 2$ generation and cell proliferation in human umbilical vein endothelial cells. Endothelium. 2008;15:175-88.

48. Zeisberg EM, Tarnavski O, Zeisberg M, Dorfman AL, McMullen JR, Gustafsson E, et al. Endothelial-to-mesenchymal transition contributes to cardiac fibrosis. Nat Med. 2007;13:952-61.

49. Becerra A, Echeverría C, Varela D, Sarmiento D, Armisén R, Nuñez-Villena F, et al. Transient receptor potential melastatin 4 inhibition prevents lipopolysaccharide-induced endothelial cell death. Cardiovasc Res. 2011;91:677-84.

50. Dimmeler S, Brinkmann S, Neugebauer E. Endotoxin-induced changes of endothelial cell viability and permeability: protective effect of a 21-aminosteroid. Eur J Pharmacol. 1995;287:257-61.
51. Fink MP. Nitric oxide synthase and vascular dysfunction in sepsis. Crit Care Med. 2014;42:1572-5.

52. Yaghi A, Paterson NA, McCormack DG. Vascular reactivity in sepsis: importance of controls and role of nitric oxide. Am J Respir Crit Care Med. 1995;151(3 Pt 1):706-12.

53. Hoover DB, Brown TC, Miller MK, Schweitzer JB, Williams DL. Loss of sympathetic nerves in spleens from patients with end stage sepsis. Front Immunol. 2017;8:1712.

54. Suzuki T, Suzuki Y, Okuda J, Kurazumi T, Suhara T, Ueda $\mathrm{T}$, et al. Sepsis-induced cardiac dysfunction and $\beta$-adrenergic blockade therapy for sepsis. J Intensive Care. 2017;5:22.

55. de Montmollin E, Aboab J, Mansart A, Annane D. Bench-tobedside review: beta-adrenergic modulation in sepsis. Crit Care. 2009; 13:230

56. Tracey KJ, Fong Y, Hesse DG, Manogue KR, Lee AT, Kuo GC, et al. Anti-cachectin/TNF monoclonal antibodies prevent septic shock during lethal bacteraemia. Nature. 1987;330:662-4.

57. Douzinas EE, Tsidemiadou PD, Pitaridis MT, Andrianakis I, Bobota-Chloraki A, Katsouyanni K, et al. The regional production of cytokines and lactate in sepsis-related multiple organ failure. Am J Respir Crit Care Med. 1997;155:53-9.

58. Pinsky MR. Clinical studies on cytokines in sepsis: role of serum cytokines in the development of multiple-systems organ failure. Nephrol Dial Transplant. 1994;9(Suppl 4):94-8.

59. Yang FL, Li CH, Hsu BG, Tsai NM, Lin SZ, Ham HJ, et al. The reduction of tumor necrosis factor-alpha release and tissue damage by pentobarbital in the experimental endotoxemia model. Shock. 2007;28:309-16.

60. Crimi E, Sica V, Slutsky AS, Zhang H, Williams-Ignarro S, Ignarro $\mathrm{LJ}$, et al. Role of oxidative stress in experimental sepsis and multisystem organ dysfunction. Free Radic Res. 2006;40:665-72.

61. Prauchner CA. Oxidative stress in sepsis: pathophysiological implications justifying antioxidant co-therapy. Burns. 2017;43:471-85.

62. Sener G, Toklu H, Kapucu C, Ercan F, Erkanil G, Kaçmaz A, et al. Melatonin protects against oxidative organ injury in a rat model of sepsis. Surg Today. 2005;35:52-9.

63. Sener G, Toklu H, Ercan F, Erkanli G. Protective effect of betaglucan against oxidative organ injury in a rat model of sepsis. Int Immunopharmacol. 2005;5:1387-96.

64. Andrades M, Ritter C, de Oliveira MR, Strek EL, Fonseca Moreira JC, Dal-Pizzol F. Antioxidant treatment reverses organ failure in rat model of sepsis: role of antioxidant enzymes imbalance, neutrophil infiltration, and oxidative stress. J Surg Res. 2011;167:e307-13. 